\title{
Offshore wind farm global blockage measured with scanning lidar
}

\author{
Jörge Schneemann ${ }^{1, \star}$, Frauke Theuer ${ }^{1, \star}$, Andreas Rott ${ }^{1}$, Martin Dörenkämper ${ }^{2}$, and Martin Kühn ${ }^{1}$ \\ ${ }^{1}$ ForWind, Institute of Physics, Carl von Ossietzky Universität Oldenburg, \\ Küpkersweg 70, 26129 Oldenburg, Germany \\ ${ }^{2}$ Fraunhofer Institute for Wind Energy Systems, Küpkersweg 70, 26129 Oldenburg, Germany \\ * These authors contributed equally to this work.
}

Correspondence: Jörge Schneemann (j.schneemann@uol.de) and Frauke Theuer

(frauke.theuer@uni-oldenburg.de)

Received: 20 November 2020 - Discussion started: 30 November 2020

Revised: 9 February 2021 - Accepted: 22 February 2021 - Published: 13 April 2021

\begin{abstract}
The objective of this paper was the experimental investigation of the accumulated induction effect of a large offshore wind farm as a whole, i.e. the global-blockage effect, in relation to atmospheric-stability estimates and wind farm operational states. We measured the inflow of a $400 \mathrm{MW}$ offshore wind farm in the German North Sea with a scanning long-range Doppler wind lidar. A methodology to reduce the statistical variability of different lidar scans at comparable measurement conditions was introduced, and an extensive uncertainty assessment of the averaged wind fields was performed to be able to identify the global-blockage effect, which is small compared to e.g. wind turbine wake effects and ambient variations in the inflow. Our results showed a $4 \%$ decrease in wind speed (accuracy range of $2 \%$ to $6 \%$ ) at transition piece height $(24.6 \mathrm{~m})$ upwind of the wind farm with the turbines operating at high thrust coefficients above 0.8 in a stably stratified atmosphere, which we interpreted as global blockage. In contrast, at unstable stratification and similar operating conditions and for situations with low thrust coefficients (i.e. approx. 0 for not operating turbines and $\leq 0.3$ for turbines operating far above rated wind speed) we identified no wind speed deficit. We discussed the significance of our measurements and possible sources of error in long-range scanning lidar campaigns and give recommendations on how to measure small flow effects like global blockage with scanning Doppler lidar. In conclusion, we provide strong evidence for the existence of global blockage in large offshore wind farms in stable stratification and the turbines operating at a high thrust coefficient by planar lidar wind field measurements. We further conclude that global blockage is dependent on atmospheric stratification.
\end{abstract}

1

Wind turbine wakes can cause negative effects at downstream turbines due to decreased wind speeds and increased turbulence (Porté-Agel et al., 2019). This was intensively studied in the last decades and is considered in all wind farm projects planned today (Rohrig et al., 2019). Recently, the so-called global-blockage effect came into the research focus. It denotes the reduction of the wind speed in a comparably wide area upstream of large wind farms. The effect is supposed to be caused by an interaction of the wind farm as a whole with the atmospheric boundary layer, since it can not be sufficiently described by a simple superposition of the induction zones of individual turbines in a large wind farm. Global blockage is usually not considered in the planning of wind energy projects and could therefore lead to a non-negligible bias in the assessment of the wind resource (Bleeg et al., 2018).

The knowledge of the wind resource to be expected during the lifetime of a wind energy project is crucial for its successful financing and economic operation. A large wind farm operator recently attributed a reduction in the predicted unlev- 
ered life cycle internal rate of return (IRR) among others to underestimated wake effects between distant wind farms and the global-wind-farm-blockage effect (Ørsted A/S, 2019).

The induction zone of a single wind turbine describes the region in front of the rotor where the wind speed is reduced due to the presence of the wind turbine. The standard for onshore power curve measurements of wind turbines recommends measuring the free wind speed, i.e. the wind speed at the turbine location in absence of the turbine, at least 2.5 rotor diameters $D$ upstream or lateral to the turbine's location (IEC, 2017). It is assumed that the influence of a wind turbine's induction zone is very low at this distance. The effect of reduced wind speeds in the induction zone of a wind turbine is called the wind turbine blockage effect, and it is caused by the thrust of the rotor. Meyer Forsting et al. (2017) give an overview of wind turbine blockage and the induction zone.

The accumulated induction zone generated by the wind farm as a whole is called global blockage and leads to a wind speed deceleration and flow deflections sideways and upward in front of the wind farm. As for solid objects in the flow like mountains or buildings, a wind farm represents an obstacle causing an upstream reverse pressure gradient which results in reduced wind speeds. Different from solid objects wind farms are porous and actively produce thrust. In the case of a wind farm, this reverse pressure gradient is referred to as global blockage. Wind-farm-related factors influencing the extent and the intensity of the global-blockage effect are wind farm size, layout, wind direction, turbine spacing and thrust coefficient (Porté-Agel et al., 2019). A meteorological parameter that affects the extent and strength of the wind farm induction zone, i.e. the global blockage, is the height of the atmospheric boundary layer (Porté-Agel et al., 2019), which is related to atmospheric stability (Kitaigorodskii and Joffre, 1988).

Knowledge about the global-blockage effect mainly results from numerical studies. Meyer Forsting et al. (2016) used RANS (Reynolds-averaged Navier-Stokes) simulations to investigate the effect that wind turbines in a row have on each other's power production when considering different inflow directions. They found a combined induction zone of the whole turbine row with changes in the individual turbine's power in the range of $-1 \%$ to $2 \%$ while the accumulated power remained nearly constant. Wu and Porté-Agel (2017) performed large-eddy simulations (LES) of large finite-size wind farms in neutral stratified boundary layers capped with a thermally stratified free atmosphere (conventionally neutral atmospheric boundary layer) and discovered a wind farm induction zone extending about $0.8 \mathrm{~km}$ (rotor diameter $D=80 \mathrm{~m}$ ) upwind and leading to power reductions of $1.3 \%$ and $3 \%$ for different farm layouts. Using LES, Allaerts and Meyers (2017) determined wind farms to excite gravity waves in stable stratification which are caused by the upward movement of the top of the boundary layer due to global wind farm blockage. Those gravity waves are similar to so-called mountain waves induced by hills and mountains.

Some modelling studies analysed global blockage from implementations of the accumulated turbine induction in engineering models. Branlard and Meyer Forsting (2020) introduced a computational inexpensive vortex model to assess wind farm production considering accumulated-blockage effects. The wind turbine and wind farm blockage effects resolved with their model compared well with results from actuator disk simulations at moderate thrust coefficients. Bleeg (2020) used a graph neural-network surrogate model to predict wind turbine interaction losses including global blockage. He found a good agreement of the model and the results of RANS simulations. Nygaard et al. (2020) coupled an engineering model for global blockage with a wind turbine wake model modified to better represent the far wind farm or cluster wake. Their wind farm blockage model was able to predict the trend in the variation of power in the front row of turbines but underestimated its amplitude. The authors pinpoint that more research is needed on the further model development and calibration. Branlard et al. (2020) presented a current overview of engineering models including global blockage and compared their performance with an actuator disk RANS simulation as a reference. They find the different models to show varying levels of accuracy with a mean error level below $1 \%$ in the induction zone.

These numerical studies agree on the magnitude of the wind speed deficit in the wind farm induction zone being in a lower one-digit percentage range. Nevertheless, most numerical studies lack measurement data for validation, since experimental investigations on global blockage have been rarely performed.

Segalini and Dahlberg (2019) measured the effect of a model wind farm on a row of upstream turbines in different distances in a laminar wind tunnel. They observed a decrease in wind speed at the turbine row in distances of up to 30 rotor diameters $(D=45 \mathrm{~mm})$ upstream and with a maximum of $2 \%$.

To our knowledge the only study presenting free-field measurements of global blockage was performed by Bleeg et al. (2018), who analysed wind measurements of meteorological masts upstream and lateral to three different onshore wind farms before and after the commercial operation date and for high thrust coefficients of the turbines. Deficits in wind speed upstream compared to the lateral reference mast of around $2 \%$, and up to more than $6 \%$ appeared typically in front of the farms after the turbines went into operation. The authors relate this mainly to the global-blockage effect.

Open-field measurements of global blockage are challenging. Classic anemometry is limited in its possibilities to study the induction zone of a wind farm, since just a limited number of masts can be placed in front of it due to mainly financial constraints. In the last decade, the remote sensing method of Doppler wind lidar (light detection and ranging) has become a common tool in many fields of wind energy 
research and applications (Hasager and Sjöholm, 2019). Lidar devices offer the possibility to scan whole wind fields with ranges of several kilometres. Commercial scanning lidar systems allow for measuring the line-of-sight (LOS) component of the wind vector on several hundred positions along the emitted laser beam and for orientating the beam in any direction. Scanning lidars have enabled many new insights into different fields of wind energy research, like wind turbine wakes (Käsler et al., 2010; Trabucchi et al., 2014), wind farm cluster wakes (Schneemann et al., 2020), resource assessment in complex terrain (Menke et al., 2020) and minutescale wind power forecasts (Theuer et al., 2020b).

The current knowledge of global blockage is mainly based on modelling activities or wind tunnel studies. Compared to well-known phenomena like wind turbine wakes with significant wind speed deficits in the order of tens of percents of the inflow wind speed in a well-defined downstream region, global blockage is much harder to study especially due to the larger spatial expansion typically over several square kilometres and the smaller wind speed differences in a single-digit percentage range. Furthermore, the effect of global blockage needs to be separated from other spatial and temporal variations in the wind field. Averaged field measurements on a small number of scattered points (Bleeg et al., 2018) lack information on superposed flow features like local wind speed variations due to orography, wind farm layout or varying meteorological conditions. Since it is not possible to distinguish these flow features, this adds uncertainty to the identification of global blockage.

Therefore, accurate field measurements spatially resolving the induction zone of the wind farm are of major importance to validate the modelling results already achieved. The extent of global blockage in operating wind farms and its dependency on atmospheric stability and the farm's operational state are still not fully understood, and proof for the effect appearing in operating wind farms is still missing.

Compact Doppler lidar systems offer the possibility to scan large parts of the inflow of a wind farm with measurement ranges up to $10 \mathrm{~km}$. Nevertheless, to obtain wind data for a quantitative analysis, all measurement parameters of the lidar device such as its orientation and tilt due to platform movements need to be carefully selected and accurately controlled. Furthermore, environmental parameters and conditions like the curvature of the earth, knowledge of the current wind profile and atmospheric stability for a height correction need to be known and accounted for.

The objective of our paper is the experimental assessment of the global-blockage effect in a large offshore wind farm dependent on atmospheric-stability estimates and wind farm operational states. In addition to this, we are proposing a method to examine comparably small flow effects like global blockage with long-range scanning lidar. Our approach includes:
- analysing horizontal long-range Doppler lidar plan position indicator (PPI) scans upstream of a $400 \mathrm{MW}$ offshore wind farm,

- deriving atmospheric stability from local meteorological measurements, and

- performing a detailed uncertainty assessment and error correction for all measured quantities.

Furthermore, we provide recommendations for measurements of global blockage or similar small flow effects with scanning Doppler lidar.

In this paper we use the terms blockage effect and wind turbine blockage effect for decreased wind speeds in the induction zone of single turbines, while we call the accumulatedblockage effect of all turbines within a wind farm or wind farm cluster, i.e. the reverse upstream pressure gradient of the wind farm, global (wind farm) blockage or the global(wind-farm-)blockage effect.

This paper is structured as follows. In Sect. 2 we introduce our experimental setup including lidar measurements and atmospheric-stability estimations. We place special focus on the uncertainty assessment of the lidar data. We present the results of four different inflow situations varying in atmospheric stability and wind speed in Sect. 3. In Sect. 4 we discuss our findings and give recommendations for lidar measurements of flow effects like global blockage with a magnitude of typical ambient wind speed fluctuations. We conclude and close the paper in Sect. 5.

\section{Methods}

In this section we describe the analysed wind farm (Sect. 2.1), lidar measurements (Sect. 2.2), meteorological measurements and atmospheric-stability characterization (Sect. 2.3), lidar data analysis (Sect. 2.4), and lidar wind speed uncertainty estimation (Sect. 2.5).

\subsection{Offshore wind farm Global Tech I}

At the time of our measurement campaign between August 2018 and January 2020, several offshore wind farms had been installed in the German and Dutch North Sea. The focus of this work is the $400 \mathrm{MW}$ wind farm "Global Tech I" (GT I). It features 80 turbines of type "Adwen AD 5-116" with a rotor diameter $D$ of $116 \mathrm{~m}$, a hub height of $92 \mathrm{~m}$, a rated power of $5 \mathrm{MW}$ at a rated wind speed of $12.5 \mathrm{~m} \mathrm{~s}^{-1}$ and a cut-in wind speed of $4 \mathrm{~m} \mathrm{~s}^{-1}$ (Global Tech I Offshore Wind $\mathrm{GmbH}, 2021$ ). Figure 1a gives an overview of the region around GT I, while Fig. 1b displays its layout. Supervisory control and data acquisition (SCADA) data of GT I were available to check the turbines status and the power production within regarded lidar scan intervals.

The "BorWin 1" cluster consisting of the operating wind farms "Veja Mate" and "BARD Offshore 1" and the wind 

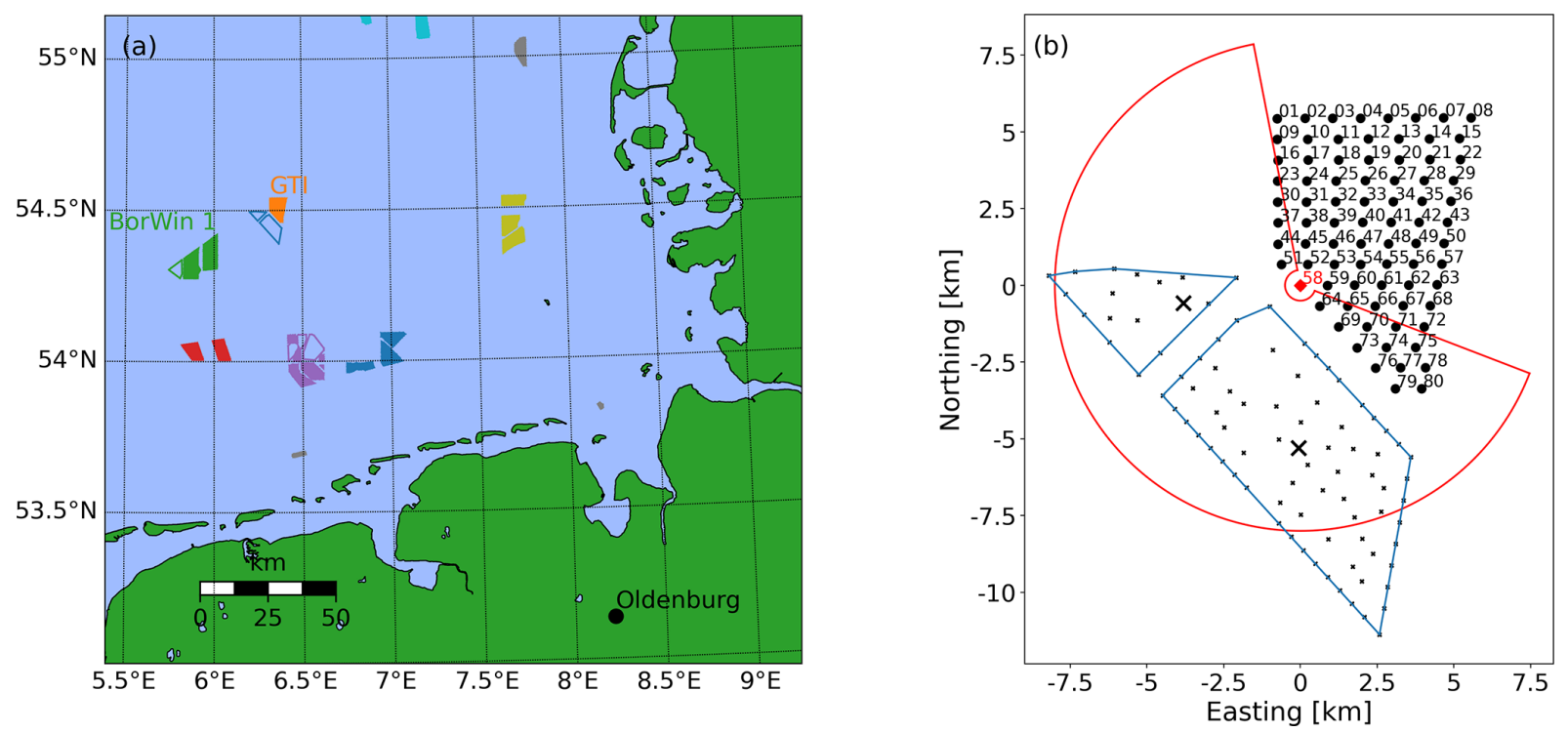

Figure 1. (a) Location of the wind farm GT I (orange) in the North Sea with neighbouring wind farms and clusters shown. Wind farms under construction are depicted as open shapes (status beginning of 2019). The $24 \mathrm{~km}$ upstream BorWin 1 cluster is marked in green. (b) Layout of GT I ( $\bullet$ with turbine numbers). The position of the lidar on turbine GT58 (red filled $\diamond)$ and the lidar scan region covering the area of the four different $150^{\circ}$ scan sectors (red line) are highlighted. The turbine locations (small $\times$ ) and the substations $(\times)$ of the wind farms Hohe See (southerly blue shape) and Albatros (northerly blue shape), which were under construction during our measurements, are marked.

farm "Deutsche Bucht" currently under construction is located at a distance of about $24 \mathrm{~km}$ in the south-west direction (green shapes in Fig. 1a). During our measurements the wind farms "Hohe See" and "Albatros" (open blue shapes in Fig. 1a and turbine coordinates in Fig. 1b) were built in the direct south-west vicinity of GT I within an approx. 1 to $6 \mathrm{~km}$ distance upstream with several transition pieces, turbines and two sub-stations installed. Measurements after the first power was fed in on 15 July 2019 were not considered (EnBW, 2019). Hard targets in the lidar data due to the construction of the two upstream wind farms Hohe See and Albatros were filtered from the measurements (cf. Sect. 2.4) and can lead to a reduced data availability on the corresponding line-of-sight direction due to (partial) laser beam coverage.

\subsection{Lidar measurements}

We used a scanning long-range Doppler wind lidar of type Leosphere Windcube 200S (serial no. WLS200S-024), which we installed on the transition piece (TP, platform to access the turbine) of turbine GT58 in GT I (red filled $\diamond$ in Fig. 1b). A photograph of the lidar on the TP is provided by Schneemann et al. (2020) in their Fig. 3. The height of the lidar's scanner was approximately $24.6 \mathrm{~m}$ a.m.s.l. (above mean sea level), $67.0 \mathrm{~m}$ below hub height and $9.0 \mathrm{~m}$ below lower-blade-tip height of the turbine. The measurement campaign started in August 2018 and ended in January 2020. We consider data from a period between February 2019 and June 2019. We performed plan position indicator (PPI) scans with an elevation of $0^{\circ}$, resulting in a measurement height of $24.6 \mathrm{~m}$ a.m.s.l. plus a correction due to the earth's curvature (up to $5 \mathrm{~m}$ at $8 \mathrm{~km}$ distance). Further, a turbine thrustdependent tilt of the lidar was observed, resulting in varying measuring heights across range gates and azimuth angles (cf. Sect. 2.5). We set the lidar's pulse length to $400 \mathrm{~ns}$, the acquisition time to $2.0 \mathrm{~s}$ and the scanning speed to $1^{\circ} \mathrm{s}^{-1}$ and scanned the upstream flow in different azimuth sectors of $150^{\circ}$ that we aligned manually to the wind direction. Range gates were defined between $500 \mathrm{~m}$ (approx. $4 \mathrm{D}$ ) and $7990 \mathrm{~m}$ (approx. $69 \mathrm{D}$ ) with a $35 \mathrm{~m}$ spacing. After intensive data filtering typical ranges around $5500 \mathrm{~m}$ were achieved (cf. Sect. 2.4). One lidar scan took $150 \mathrm{~s}$ and resulted in 215 range gates (also referred to as "measurement points") on each of the 75 beams. The further processing of the lidar scans is described in Sect. 2.4. Schneemann et al. (2020) give further information about the measurements, and Schneemann et al. (2019) provide some exemplary lidar scans from this campaign.

\subsection{Atmospheric-stability characterization and meteorological measurements}

For the analysis of the global-blockage effect knowledge about wind speed at one common height across the whole scan is required. The varying measuring height, as a consequence of the tilt of the lidar device and the earth's curvature, thus necessitates the extrapolation of wind speeds to that common height. To keep extrapolation distances small, we here chose the height of the transition piece or lidar device. For the extrapolation we use a stability-corrected loga- 
rithmic wind profile. Information regarding stability further allows us to analyse the effect of atmospheric conditions on global blockage.

We used a similar methodology to derive atmospheric stratification as in Theuer et al. (2020b) and Schneemann et al. (2020), which is described here for completeness. To characterize atmospheric stability (Emeis, 2018), we used local measurements as well as reanalysis data. On the transition piece of turbine GT58 close to the lidar's position, we measured air temperature and humidity (Vaisala HMP155) and air pressure (Vaisala PTB330). Additionally, we used the sea surface temperature (SST) from the OSTIA data set (Operational Sea Surface Temperature and Ice Analysis; Good et al., 2020). We utilized a methodology introduced by Rodrigo et al. (2015) to estimate the bulk Richardson number of

$\mathrm{Ri}_{\mathrm{b}}=\frac{g}{T_{\mathrm{v}}} \frac{0.5 z_{\mathrm{TP}}\left(\Theta_{\mathrm{TP}}-\Theta_{0}\right)}{u_{\mathrm{li}}^{2}}$,

where, $g$ is the gravitational acceleration, $T_{\mathrm{v}}$ is the virtual temperature at sea level, and $\Theta_{\mathrm{TP}}$ and $\Theta_{0}$ are the virtual potential temperatures at TP height $z_{\text {TP }}$ and sea level, respectively. $u_{\mathrm{li}}$ describes the wind speed at the lidar position, determined utilizing lidar measurements up to range gates of $600 \mathrm{~m}$. The height used to calculate $\mathrm{Ri}_{\mathrm{b}}$ is defined as the mean between the two height levels, i.e. $0.5 z_{\text {TP }}$. After estimating $\mathrm{Ri}_{\mathrm{b}}$ we obtain the dimensionless stability parameter of

$\zeta= \begin{cases}\frac{10 \mathrm{Ri}_{\mathrm{b}}}{1-5 \mathrm{Ri}_{\mathrm{b}}} & \mathrm{Ri}_{\mathrm{b}}>0 \\ 10 \mathrm{Ri}_{\mathrm{b}} & \mathrm{Ri}_{\mathrm{b}} \leq 0,\end{cases}$

and finally the Obukhov length of

$L=\frac{0.5 z_{\mathrm{TP}}}{\zeta}$.

We estimated the roughness length $z_{0}$ using the determined Obukhov length $L$ and the stability-corrected logarithmic wind profile of

$u=\sqrt{\frac{z_{0} g}{\alpha_{c}}} \frac{1}{\kappa}\left(\ln \left(\frac{z}{z_{0}}\right)-\Psi\left(\frac{z}{L}\right)\right)$,

with $z=z_{\mathrm{TP}}$ and $u=u_{\mathrm{TP}}$. Here, $\kappa=0.4$ describes the von Kármán constant, and $\alpha_{\mathrm{c}}=0.011$ describes the Charnock parameter, often used in an offshore context (Smith, 1980). The stability correction term of

$\Psi= \begin{cases}2 \ln \left(\frac{1+x}{2}\right)+\ln \left(\frac{1+x^{2}}{2}\right)-2 \arctan (x)+\frac{\pi}{2} & L<0 \\ -\beta \frac{z}{L} & L \geq 0\end{cases}$

was defined following Dyer (1974) with $x=\left(1-\gamma \frac{z}{L}\right)^{1 / 4}$, $\gamma=19.3$ and $\beta=6$ (Högström, 1988).

\subsection{Lidar data processing}

We filtered the lidar scans using a carrier-to-noise (CNR) threshold filter, considering only values with $-26 \mathrm{~dB}<\mathrm{CNR}<0 \mathrm{~dB}$. With a velocity-azimuth display (VAD) algorithm, we calculated a mean wind speed $\bar{u}$ and wind direction $\chi$ individually for each scan assuming a homogeneous wind field and neglecting the vertical wind speed component (Werner, 2005). At each measurement point we projected the line-of-sight (LOS) wind velocities $u_{\text {LOS }}$ onto the mean wind direction by means of

$u_{\mathrm{h}}=\frac{u_{\mathrm{LOS}}}{\cos (\vartheta-\chi)}$,

with horizontal wind speed $u_{\mathrm{h}}$ and azimuth angles $\vartheta$. Sectors with azimuth angles almost perpendicular to the wind direction, i.e.

$75^{\circ}<|\vartheta-\chi|<105^{\circ}$,

were neglected, as they are associated with large errors. Further, outliers with $\left|u_{\mathrm{h}}-\bar{u}\right|>2.75 \sigma_{\mathrm{u}}$, with $\sigma_{\mathrm{u}}$ defined as the standard deviation of horizontal wind speed within each scan, were discarded. For each measurement point we then determined the measuring height, considering both the curvature of the earth and a turbine thrust-dependent tilt of the lidar device. Further details about the alignment of the lidar and the correction function to estimate the measurement height are presented in Rott et al. (2021). After assessing the measuring height, wind speed values were extrapolated to the lidar height $z_{\mathrm{TP}}=24.6 \mathrm{~m}$ using a stability-corrected logarithmic wind speed profile described by Eqs. (4) and (5) (Sect. 2.3).

Only scans with a data availability of at least $60 \%$ were considered for further analysis. Data availability was calculated individually for each scan, including measurement points up to a range gate of $7000 \mathrm{~m}$ and not considering critical sectors as defined in Eq. (7).

Finally, we interpolated all valid scans to a Cartesian grid with a spacing of $\Delta x=\Delta y=50 \mathrm{~m}$ to be able to average data of varying scanning sectors.

For further analysis, the lidar scans were categorized according to their respective mean wind direction $\chi, 10 \mathrm{~min}$ mean wind speed measured by a sonic anemometer at the nacelle of turbine GT58 $\bar{u}_{\text {GT58 }}$ and atmospheric stability characterized by $L$. In each category, consisting of $N$ individual lidar scans $i$, we performed the following steps: first, the mean wind speed within the scan $i$ at TP height $\bar{u}_{\mathrm{TP}, i}$ was derived and used to normalize the wind speeds on all grid points, yielding $u_{\mathrm{TP}}$,norm, $i$. Second, all normalized scans were averaged to $\bar{u}_{\text {TP,norm. }}$. Hereby, Cartesian grid points with data availability $<80 \%$, i.e. $N_{\mathrm{r}}<0.8 N$, with $N$ being the number of all available scans within the category and $N_{\mathrm{r}}$ being the number of valid scans at each grid point, were neglected. Third, normalized and averaged wind speeds $\bar{u}_{\text {TP,norm }}$ were interpolated onto a virtual line in the mean wind direction upstream of the lidar. 
Table 1. Summary of possible scenarios to be analysed. Those shown in this work are named; those not shown are marked as $X$.

\begin{tabular}{lll}
\hline & Unstable $(L<0 \mathrm{~m}$ and $|L|<1000 \mathrm{~m})$ & Stable $(L>0 \mathrm{~m}$ and $|L|<1000 \mathrm{~m})$ \\
\hline Not operating & $X$ & Scenario 2, Fig. 5 \\
Operating below rated wind speed & Scenario 1, Fig. 4 & Scenario 4, Fig. 7 \\
Operating above rated wind speed & $X$ & Scenario 3, Fig. 6 \\
\hline
\end{tabular}

For the blockage analysis we decided to distinguish between two stability classes, i.e. unstable and stable situations, and three different operational states, respectively, for wind speed ranges. The operational state of the wind farm was estimated using SCADA power data and the wind speed range based on the wind speed at nacelle height. These states are namely the wind farm not operating (below cut-in wind speed, low thrust coefficient of approx. 0), the wind farm operating at rated power (above rated wind speed, low to moderate thrust coefficient) and the wind farm operating below and up to rated power (below rated wind speed, high thrust coefficient). In total, the combination of these two categories left us with a number of six possible cases to be analysed as summarized in Table 1.

However, for brevity we omitted the combinations unstable, not operating and unstable, operating above rated wind speed. With the comparison of the four remaining cases we aimed to cover both scenarios where global blockage is likely to occur and those where an occurrence is less likely. This "cross-check" allowed us to better interpret the obtained results in terms of possible wind speed gradients caused by other background phenomena. We start with the analysis of the remaining unstable scenario and then continue with the stable cases, sorted according to their thrust coefficients. The four scenarios are summarized below.

- Scenario 1: wind turbines operating below and up to rated power with a high thrust coefficient $>0.8$ at the plateau region of the thrust-coefficient curve. We chose a wind speed interval of $8 \mathrm{~ms}^{-1}<\bar{u}<11 \mathrm{~m} \mathrm{~s}^{-1}$, unstable atmospheric conditions and a total power production of the wind farm with at least $50 \%$ of the wind farm's rated power and $80 \%$ of the wind farm's estimated power. Here, the wind farm power is estimated by extrapolating $u_{\mathrm{TP}}$ to hub height using an average logarithmic profile (see Sect. 2.3, with $L=-300 \mathrm{~m}$ ) and transferring the result to the whole wind farm considering wind farm effects. Further, only situations with high power production at GT58 $\left(P_{\mathrm{GT} 58} \geq 4000 \mathrm{~kW}\right)$ were considered to make the experienced tilt of the lidar device comparable to that of Scenario 4.

- Scenario 2: wind farm not operating with wind speeds below cut-in wind speed, i.e. thrust coefficients of approx. 0. Here scans with wind speed $u_{\mathrm{TP}}$ from $3 \mathrm{~ms}^{-1}<\bar{u}<4 \mathrm{~ms}^{-1}$, during stable atmospheric con- ditions and a wind farm power production $<5 \%$ of the wind farm's rated power were selected.

- Scenario 3: wind farm operating at rated power with wind speeds $u_{\mathrm{TP}}$ above rated wind speed and low thrust coefficients $\leq 0.3$. This comprises scans with $16 \mathrm{~m} \mathrm{~s}^{-1}<\bar{u}<22 \mathrm{~m} \mathrm{~s}^{-1}$, stable atmospheric conditions and a total wind farm power $>80 \%$ of the rated power. Further, only cases with a blade pitch from SCADA data $>5^{\circ}$ at GT58 were considered.

- Scenario 4: wind turbines operating below and up to rated power with a high thrust coefficient $>0.8$. This is the same as Scenario 1; however here we chose scans with stable atmospheric conditions. In this case the estimated wind farm power was determined using an average logarithmic profile with $L=300 \mathrm{~m}$.

\subsection{Uncertainty estimation}

For the further analysis and interpretation of the results, several uncertainties introduced in the course of the measurement campaign and data analysis procedure are important to consider. In this section, we qualitatively summarize the most important error contributions and subsequently estimate uncertainties using three different methodologies. First, we calculate the total propagated uncertainty using the uncertainties assigned to the individual components with Gaussian error propagation; second, we determine the total propagated uncertainty as before but distinguish also between range-gateindependent and range-gate-correlated input variables; and third, we derive the statistical standard error of the mean.

We summarize sources of errors and uncertainties that need to be considered in offshore lidar measurements of flow effects with small deviations with respect to the mean flow in Table 2 and visualize them in Fig. 2. It becomes clear that several of the error sources are directly or indirectly linked to the alignment of the lidar: the device's tilt causes the need for a height extrapolation; thus wind profile information is required, introducing additional uncertainties. Considering a measurement scenario with perfect horizontal measurements, the error sources could be significantly reduced. However, as in this setup an extrapolation of wind speed $u_{\mathrm{m}}$ at measuring height $z_{\mathrm{m}}$ to lidar height $z_{\mathrm{TP}}$ is required, we estimated the uncertainty associated with it in more detail in the following. 


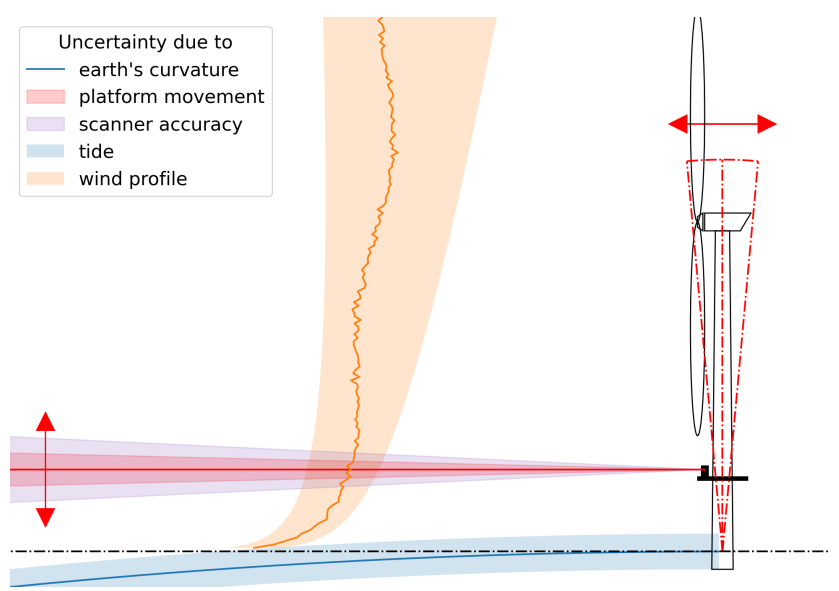

Figure 2. Illustration of different sources of uncertainty for wind speed estimates in long-range Doppler lidar measurements on an offshore platform like the TP of an offshore wind turbine. Besides the general uncertainty in the LOS wind speed measurement the main source of uncertainty is the varying measurement height due to lidar scanner misalignment (purple) and platform tilts and movements (red), e.g. due to the turbine's thrust. Curvature of the earth (blue) and tide (light blue) add to the height uncertainty. As a consequence of known height errors measured wind speeds need to be transformed back to the desired height; thus the lack of knowledge of the prevailing wind profile introduces additional uncertainty (orange).

\subsubsection{Total propagated uncertainty}

As stated earlier the height extrapolation of lidar data is performed by means of a stability-corrected logarithmic wind speed profile (Eq. 4). The wind speed at height of the TP $u_{\mathrm{TP}}$ can thus be expressed as

$u_{\mathrm{TP}}=u_{\mathrm{m}} \frac{\ln \left(\frac{z_{\mathrm{TP}}}{z_{0}}\right)-\Psi\left(\frac{z_{\mathrm{TP}}}{L}\right)}{\ln \left(\frac{z_{\mathrm{m}}}{z_{0}}\right)-\Psi\left(\frac{z_{\mathrm{m}}}{L}\right)}$.

Gaussian error propagation yields the total propagated uncertainty of

$$
\begin{aligned}
\Delta u_{\mathrm{TP}}= & {\left[\left(\frac{\ln \left(\frac{z_{\mathrm{TP}}}{z_{0}}\right)-\Psi_{\mathrm{TP}}}{\ln \left(\frac{z_{\mathrm{m}}}{z_{0}}\right)-\Psi_{\mathrm{m}}} \Delta u_{\mathrm{m}}\right)^{2}\right.} \\
& +\left(\frac{u_{\mathrm{m}}\left(\ln \left(\frac{z_{\mathrm{TP}}}{z_{0}}\right)-\Psi_{\mathrm{TP}}+\Psi_{\mathrm{m}}-\ln \left(\frac{z_{\mathrm{m}}}{z_{0}}\right)\right)}{z_{0}\left(\ln \left(\frac{z_{\mathrm{m}}}{z_{0}}\right)-\Psi_{\mathrm{m}}\right)^{2}} \Delta z_{0}\right)^{2} \\
& +\left(\frac{u_{\mathrm{m}}\left(\Psi_{\mathrm{TP}}-\ln \left(\frac{z_{\mathrm{TP}}}{z_{0}}\right)\right)}{z_{\mathrm{m}}\left(\ln \left(\frac{z_{\mathrm{m}}}{z_{0}}\right)-\Psi_{\mathrm{m}}\right)^{2}} \Delta z_{\mathrm{m}}\right)^{2} \\
& +\left(\frac{u_{\mathrm{m}}}{\Psi_{\mathrm{m}}-\ln \left(\frac{z_{\mathrm{m}}}{z_{0}}\right)} \Delta \Psi_{\mathrm{TP}}\right)^{2} \\
& \left.+\left(\frac{u_{\mathrm{m}}\left(\ln \left(\frac{z_{\mathrm{TP}}}{z_{0}}\right)-\Psi_{\mathrm{TP}}\right)}{\left(\ln \left(\frac{z_{\mathrm{m}}}{z_{0}}\right)-\Psi_{\mathrm{m}}\right)^{2}} \Delta \Psi_{\mathrm{m}}\right)^{2}\right]^{1 / 2}
\end{aligned}
$$

with the corresponding uncertainty in the stability correction term of

$$
\Delta \Psi= \begin{cases}\left|\frac{4 x^{2}}{x^{3}+x^{2}+x+1}\right| \Delta x & L<0 \\ {\left[\left(-\beta \frac{1}{L} \Delta z\right)^{2}+\left(\beta \frac{z}{L^{2}} \Delta L\right)^{2}\right]^{1 / 2}} & L \geq 0\end{cases}
$$

where

$$
\begin{aligned}
\Delta x & =\left[\left(-\frac{\gamma}{4 L}\left(1-\gamma \frac{z}{L}\right)^{-3 / 4} \Delta z\right)^{2}\right. \\
& \left.+\left(\frac{\gamma z}{4 L^{2}}\left(1-\gamma \frac{z}{L}\right)^{-3 / 4} \Delta L\right)^{2}\right]^{1 / 2} .
\end{aligned}
$$

The indices of the correction terms $\Psi$ refer to the height at which it is determined. The uncertainty of the Obukhov length $L$ is also determined by means of Gaussian error propagation of Eqs. (1) to (3), leading to

$$
\begin{aligned}
& \Delta \mathrm{Ri}_{\mathrm{b}}= {\left[\left(\frac{-g}{T_{\mathrm{v}}^{2}} \frac{0.5 z_{\mathrm{TP}}\left(\Theta_{\mathrm{TP}}-\Theta_{0}\right)}{u_{\mathrm{li}}^{2}} \Delta T_{\mathrm{v}}\right)^{2}\right.} \\
&+\left(\frac{-2 g}{T_{\mathrm{v}}} \frac{0.5 z_{\mathrm{TP}}\left(\Theta_{\mathrm{TP}}-\Theta_{0}\right)}{u_{\mathrm{li}}^{3}} \Delta u_{\mathrm{li}}\right)^{2} \\
&+\left(\frac{g}{T_{\mathrm{v}}} \frac{0.5 z_{\mathrm{TP}}}{u_{\mathrm{li}}^{2}} \Delta \Theta_{0}\right)^{2} \\
&\left.+\left(\frac{g}{T_{\mathrm{v}}} \frac{0.5 z_{\mathrm{TP}}}{u_{\mathrm{li}}^{2}} \Delta \Theta_{\mathrm{TP}}\right)^{2}\right]^{1 / 2}, \\
& \Delta \zeta= \begin{cases}\left|\frac{10}{\left(1-5 \mathrm{Ri}_{\mathrm{b}}\right)^{2}}\right| \Delta \mathrm{Ri}_{\mathrm{b}} & \mathrm{Ri}_{\mathrm{b}}>0 \\
10 \Delta \mathrm{Ri}_{\mathrm{b}} & \mathrm{Ri}_{\mathrm{b}} \leq 0,\end{cases} \\
& \Delta L=\left|\begin{array}{ll}
-0.5 z_{\mathrm{TP}} \\
\zeta^{2}
\end{array}\right| \Delta \zeta .
\end{aligned}
$$

The uncertainties $\Delta T_{\mathrm{v}}, \Delta \Theta_{0}$ and $\Delta \Theta_{\mathrm{TP}}$ are hereby assessed using air and water temperature, humidity, and pressure uncertainties. We set $\Delta T_{\text {air }}=0.1 \mathrm{~K}, \Delta T_{\text {water }}=0.2 \mathrm{~K}$, $\Delta p=0.3 \mathrm{hPa}$ and $\Delta H=1.2 \%$, following typical uncertainties suggested in the sensors' user manuals.

Other uncertainty contributions are set to $\Delta u_{\mathrm{li}}=0.1 \mathrm{~m} \mathrm{~s}^{-1}$ and $\Delta z_{0}=0.05 z_{0}$. The wind speed uncertainty at measuring height $\Delta u_{\mathrm{m}}$ is dependent on the line-of-sight wind speed uncertainty $\Delta u_{\mathrm{LOS}}=0.1 \mathrm{~ms}^{-1}$, the azimuth uncertainty $\Delta \vartheta=0.05^{\circ}$ and the wind direction uncertainty $\Delta \chi=1^{\circ}$, following error propagation of Eq. (6). $\Delta z_{\mathrm{m}}$ was estimated using the pitch and roll uncertainty, which were set to $\Delta \beta=$ $\Delta \gamma=0.05^{\circ}$ following the findings of the method of sea surface levelling demonstrated in Rott et al. (2017, 2021). These uncertainties can be understood to comprise both possible elevation pointing uncertainties as well as the tilt of the lidar device. All uncertainty terms defined here and thus also 
Table 2. Possible errors and uncertainties that might be introduced during the lidar measurement campaign and data analysis. In addition to a description of the uncertainty components, the measures we take to minimize those are stated, and it is indicated whether they are considered in the uncertainty estimation.

\begin{tabular}{|c|c|c|}
\hline Uncertainty components & Description & Measures \\
\hline Azimuth or elevation pointing error & Internal unknown offsets of lidar scanner & $\begin{array}{l}\text { Not corrected for, calibration prior to mea- } \\
\text { surement campaign recommended; consid- } \\
\text { ered in uncertainty estimation }\end{array}$ \\
\hline Movement or tilt of lidar & $\begin{array}{l}\text { Uncertainties in pointing accuracy, in par- } \\
\text { ticular caused by a wind turbine thrust- } \\
\text { dependent tilt of the device; influences } \\
\text { the measurement height of the device and } \\
\text { varies with the range gate }\end{array}$ & $\begin{array}{l}\text { Empirical correction function for thrust- } \\
\text { dependent platform movement (Rott et al., } \\
\text { 2021); considered in uncertainty estima- } \\
\text { tion }\end{array}$ \\
\hline
\end{tabular}

Curvature of the earth

Tide

Uncertainty in elevation angle due to the earth's curvature, lidar tilt and scanner pointing error

LOS wind speed

Assumption of a homogeneous wind field

Uncertainties in meteorological measurements

Uncertainties in roughness length estimation

Inapplicability of the logarithmic wind profile

Laser beam deflection due to thermal gradients in the lower boundary layer
Systematic variation in measuring height

Uncertainty in measuring height estimated to be $\pm 0.6 \mathrm{~m}$ here

Transfer of tilted LOS wind speed to horizontal wind speed $(1 / \cos )$

Uncertainty in LOS wind speed causes uncertainty in horizontal wind speed

Wind speed error as a consequence of wind field reconstruction (VAD algorithm)

Results in uncertainties in stability estimation

Results in uncertainties in wind profile estimation

Occurs especially during stable atmospheric conditions (Theuer et al., 2020a; Peña et al., 2008), which might be related to the occurrence of e.g. kinks or low-level jets (Møller et al., 2020); leads to uncertainty in wind speed correction to lidar height

Results in measurement height error
Corrected for, not considered in uncertainty estimation

Not corrected for, not considered in uncertainty estimation

Here less then $1^{\circ}$ in total, contribution neglectable; not corrected for, not considered in uncertainty estimation

Not corrected for, considered in uncertainty estimation

Not corrected for, not considered in uncertainty estimation

Not corrected for, considered in uncertainty estimation

Not corrected for, considered in uncertainty estimation

Not corrected for, not considered in uncertainty estimation

Not corrected for, not considered in uncertainty estimation the total propagated uncertainty $\Delta u_{\mathrm{TP}}$ are understood as the $1.96 \sigma$ values of the corresponding error distributions; i.e. we expect them to include $95 \%$ of all values.

A detailed analysis of the uncertainty associated with wind speed extrapolation to hub height in the framework of an offshore lidar campaign by Theuer et al. (2020a) has revealed a strong dependency on the Obukhov length $L$. Large uncertainties need to be expected especially during very stable atmospheric conditions. Even though the study uses different input parameters, this also holds valid for our analysis.

We determined the total propagated uncertainty $\Delta u_{\mathrm{TP}}$ for each scan and grid point. Values were normalized within each scan $i$ using $\bar{u}_{\mathrm{TP}, i}$ and averaged across all valid scans, yielding $\overline{\Delta u}_{\mathrm{TP} \text {,norm }}$.

\subsubsection{Corrected propagated uncertainty}

In the uncertainty estimation of the total propagated uncertainty (Sect. 2.5.1) we defined $\overline{\Delta u}_{\mathrm{TP} \text {,norm }}$ in a way that assumes none of the input uncertainties are correlated across range gates. That means we also assume it is possible that the signs of the errors vary between range gates. While this might be true for wind speed errors $\Delta u_{\mathrm{m}}$ and roughness length errors $\Delta z_{0}$, it does not hold for measurement height errors $\Delta z_{\mathrm{m}}$, which are directly related to the tilt of the lidar, and the Obukhov length error $\Delta L$, which we consider to be constant across the whole measurement domain. Since these assumptions could influence the interpretation of the results, we decided to determine the uncertainty additionally only considering measurement-range-independent input variables. That means we set $\Delta z_{\mathrm{m}}=\Delta \Psi_{\mathrm{TP}}=\Delta \Psi_{\mathrm{m}}=0$ to calculate the cor- 
rected propagated uncertainty as

$$
\begin{aligned}
& \Delta u_{\mathrm{TP}, \mathrm{cor}}=\left[\left(\frac{\ln \left(\frac{z_{\mathrm{TP}}}{z_{0}}\right)-\Psi_{\mathrm{TP}}}{\ln \left(\frac{z_{\mathrm{m}}}{z_{0}}\right)-\Psi_{\mathrm{m}}} \Delta u_{\mathrm{m}}\right)^{2}\right. \\
& \left.+\left(\frac{u_{\mathrm{m}}\left(\ln \left(\frac{z_{\mathrm{TP}}}{z_{0}}\right)-\Psi_{\mathrm{TP}}+\Psi_{\mathrm{m}}-\ln \left(\frac{z_{\mathrm{m}}}{z_{0}}\right)\right)}{z_{0}\left(\ln \left(\frac{z_{\mathrm{m}}}{z_{0}}\right)-\Psi_{\mathrm{m}}\right)^{2}} \Delta z_{0}\right)^{2}\right]^{1 / 2} .
\end{aligned}
$$

Also $\Delta u_{\text {TP,cor }}$ is normalized within each scan and subsequently averaged across all valid scans to $\overline{\Delta u}_{\mathrm{TP}}$,cor .

We examine the uncertainty contributions of $\Delta z_{\mathrm{m}}, \Delta \Psi_{\mathrm{TP}}$ and $\Delta \Psi_{\mathrm{m}}$ for relevant cases separately in a case distinction in Sect. 3.4.

\subsubsection{Standard error of the mean}

As an alternative to the total propagated uncertainty we calculated the statistical error, i.e. the standard error of the mean, as

$\mathrm{SEM}=1.96 \frac{\sigma_{u_{\mathrm{TP}, \mathrm{norm}}}}{\sqrt{N_{\mathrm{r}}}}$

for each grid point, considering all valid scans $N_{\mathrm{r}}$ with the standard deviation of the normalized wind speed at each grid point $\sigma_{u_{\mathrm{TP} \text {,norm }}}$. We included the factor 1.96 already in the definition of the variable to cover the $95 \%$ confidence interval for normally distributed errors. The SEM estimates the deviation of the sample mean from the true mean (McKillup, 2005) and thus yields information regarding the statistical significance of the results. While the total propagated uncertainty regards the accuracy of single input variables, the statistical error quantifies the precision of the results from different scans. A higher number of scans typically reduces measurement noise from the statistical error, i.e. wind speed fluctuations around the mean.

\subsubsection{Uncertainty due to local wind direction deviations}

In addition to the total propagated uncertainty, the corrected propagated uncertainty and the SEM, which focus on the uncertainty in the mean wind direction, we want to introduce another uncertainty into the measured wind fields that arises from the assumption of a homogeneous wind direction in the whole scanned area. As described in Sect. 2.4 we estimate the mean wind direction of a single lidar scan by means of a cosine fit (VAD algorithm) and transfer all measured line-ofsight wind speeds of the current scan to absolute horizontal wind speeds using this fixed mean wind direction (Eq. 6). Local deviations from this mean wind direction lead to estimation errors of the horizontal wind. This uncertainty contribution is dependent on the angular difference between the wind direction and the lidar scanner's azimuth angle and the degree of the deviation. To quantify this, we define the local

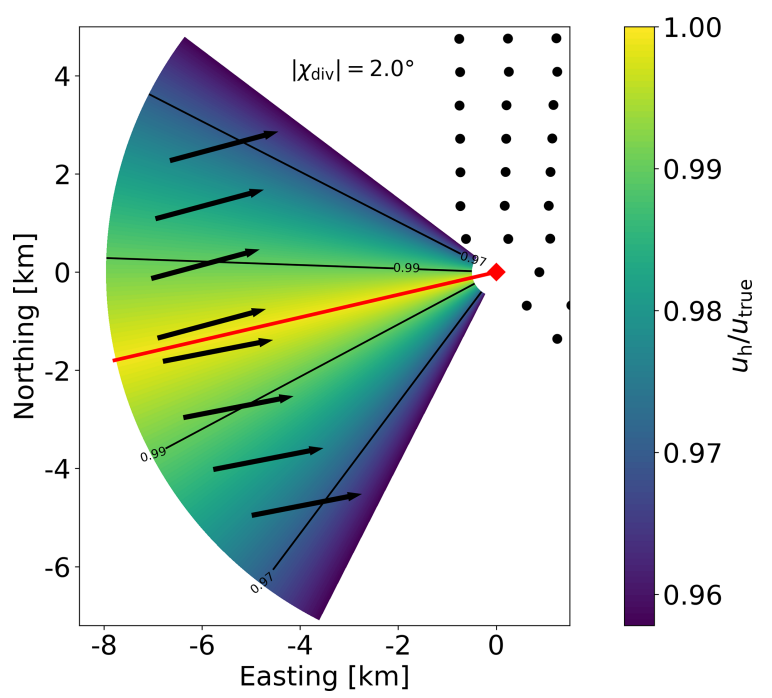

Figure 3. Local deviation in the assessment of the horizontal wind speed $u_{\mathrm{h}}$ due to a wind direction deviation $\left|\chi_{\text {div }}\right|$, here exemplarily set to $2.0^{\circ}$ (cf. Eq. 16). The lidar (red $\diamond$ ) directs its beam (red) in the mean wind direction $\chi$; arrows denote the local deviation of the wind direction $\chi_{\text {div }}$.

uncertainty as

$\frac{u_{\mathrm{h}}}{u_{\text {true }}}=\frac{\frac{u_{\mathrm{LOS}}}{\cos (\vartheta-\chi)}}{\frac{u_{\mathrm{LOS}}}{\cos \left(\vartheta-\chi_{\text {true }}\right)}}=\frac{\cos \left(\vartheta-\chi_{\text {true }}\right)}{\cos (\vartheta-\chi)}$

with the local estimated wind speed $u_{\mathrm{h}}$ and the true local wind speed $u_{\text {true }}$ that considers wind direction deviations being dependent on the lidar's azimuth angle $\vartheta$ and the local wind direction $\chi_{\text {true }}=\chi+\chi_{\text {div }}$ being the sum of the mean wind direction $\chi$ and the local wind direction deviation $\chi_{\text {div }}$.

Figure 3 visualizes this error for a hypothetical lidar scan with a constant deviation of $\left|\chi_{\operatorname{div}}\right|=2.0^{\circ}$ and its sign chosen corresponding to a flow around the wind farm. While the error vanishes for the lidar looking upwind, it leads to reduced wind speed estimates of more than $4 \%$ at the sides of the scan $\left(\vartheta-\chi= \pm 50^{\circ}\right)$ in this example. Since this uncertainty contribution is on the same order of magnitude as the expected strength of global blockage at the sides of the scan, we focus our analysis on the upstream direction where the error is neglectable.

\section{Results}

In the following we present results of the four scenarios introduced in Sect. 2.4.

\subsection{Scenario 1: wind farm operating below rated wind speed at unstable atmospheric conditions with a high thrust coefficient}

Figure 4a shows $N=53$ normalized and averaged lidar scans for unstable atmospheric conditions and within a wind speed 

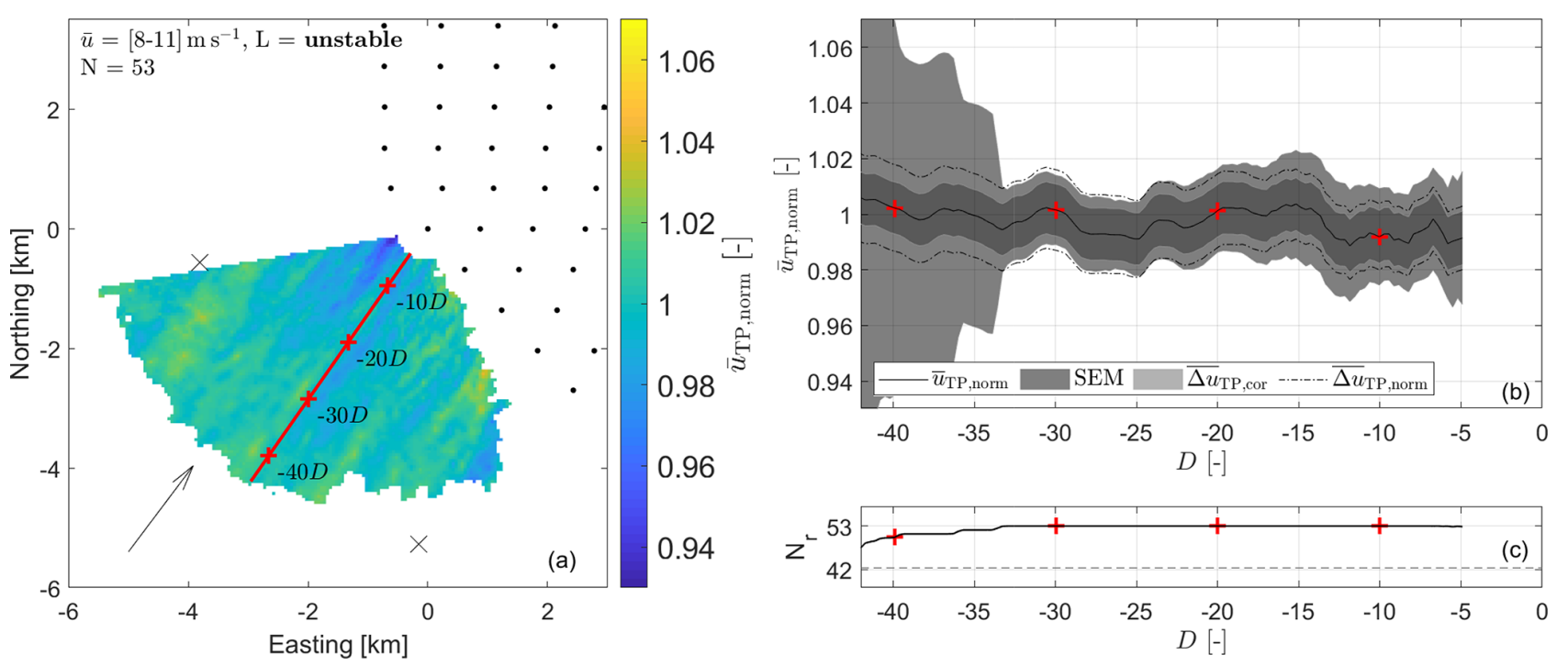

Figure 4. Scenario 1: analysis of lidar scans during unstable atmospheric conditions and in cases with a high thrust coefficient with $\bar{u}=[8-$ 11] $\mathrm{ms}^{-1}, \chi=[210-220]^{\circ}, N=53, \bar{\chi}=217^{\circ}, \bar{u}_{\mathrm{GT} 58}=10.11 \mathrm{~m} \mathrm{~s}^{-1}, \bar{P}_{\mathrm{GT} 58}=4.77 \mathrm{MW}$, the median Obukhov length $L_{\mathrm{med}}=-430 \mathrm{~m}$ and the standard deviation of the wind direction $\sigma_{\chi}=2.68^{\circ}$. Panel (a) depicts the normalized wind speed $\bar{u}_{\text {TP,norm }}$ averaged over all valid scans $N$. The arrow displays the mean wind direction $\bar{\chi} \cdot \bar{u}_{\text {TP,norm }}$ along the wind field cut, indicated as a red line in (a), is shown in (b). Here, additionally the three estimated uncertainties $\overline{\Delta u}_{\text {TP,cor }}$, SEM and $\overline{\Delta u}_{\text {TP,norm }}$ are visualized as grey-shaded areas and a black dotted line, respectively. Be aware that the SEM here overlays $\overline{\Delta u}$ TP,cor. The distance to the lidar on the $x$ axis is given in terms of rotor diameter $D$. Panel (c) displays the number of valid scans at each grid point $N_{\mathrm{r}}$. The grey horizontal dashed line marks $0.8 N$. Points highlighted by a red + in (b) and (c) correspond to the locations marked in the lidar cut in (a) and (b).

interval of $\bar{u}=[8-11] \mathrm{m} \mathrm{s}^{-1}$, i.e. for the wind farm operating at a high thrust coefficient. The wind field depicted is relatively homogeneously across the shown area, with slight variations of wind speed visible as streaks in the wind direction. Apart from that, most values fluctuate closely around the mean wind speed.

This impression is confirmed by Fig. 4b, where a virtual cut in mean wind direction upstream of the lidar, indicated as a red line in Fig. $4 \mathrm{a}$, is depicted. Again, $\bar{u}_{\mathrm{TP}}$,norm fluctuates around 1, and the three error terms (cf. Eqs. 9, 14 and 15), visualized as light- and dark-grey-shaded areas and a black dotted line, respectively, have a similar magnitude. Be aware that the SEM overlays the corrected propagated uncertainty $\overline{\Delta u}_{\text {TP,cor }}$ and makes its shaded area seem darker than depicted in the legend. The uncertainty contributions of $L$ and $z_{\mathrm{m}}$ are relatively low; i.e. $\overline{\Delta u}_{\mathrm{TP}}$,norm is only slightly larger than $\overline{\Delta u}_{\text {TP,cor }}$. This can be attributed to the relatively small change of wind speed with height during unstable conditions. Generally slightly larger values can be observed for the SEM as compared to $\overline{\Delta u}_{\mathrm{TP}, \text { cor }}$. For far range gates from approximately $-33 D$ onward, the SEM increases significantly as a consequence of lower data quality and the lower number of values considered here (cf. Fig. 4c). We found no evidence for a decreasing trend in wind speed upstream of the wind farm GT I for Scenario 1.

\subsection{Scenario 2: wind farm not operating at stable atmospheric conditions}

Figure 5 shows normalized and averaged wind speeds in the inflow region of GT I for stable atmospheric conditions and wind speeds below cut-in in the same manner as Fig. 4. Strong relative variations of $\bar{u}_{\mathrm{TP} \text {,norm }}$ from the average wind speed are visible across the scan. Larger values occur for low azimuth angles, i.e. south of the wind farm. Two wakes, located on both sides of the wind field cut, with diminished wind speed are visible. These wakes are likely caused by jack-up barges used to construct the two neighbouring wind farms. Despite the large wind speed fluctuations within the scan area, no decrease of wind speed with decreasing distance to the wind farm can be observed, in neither Fig. 5a nor $b$. The measurements show a slight increase in the wind speed of approximately $3 \%$ up to approximately $-30 \mathrm{D}$ and decrease again closer to the wind farm. One should be aware that due to the low average wind speed ranging from $\bar{u}=3-$ $4 \mathrm{~m} \mathrm{~s}^{-1}$ with a mean value of $\bar{u}_{\mathrm{TP}}=3.48 \mathrm{~m} \mathrm{~s}^{-1}$ the large percental variation in wind speed across the scan is low in absolute values.

Especially distinct are the large values of $\overline{\Delta u}_{\text {TP,norm }}$ shown in Fig. 5b. We attribute this to the very stable atmospheric conditions, which cause large wind speed extrapolation uncertainties (Theuer et al., 2020a) and low wind speeds, which result in larger relative uncertainties. Be aware of the different $y$ scale as compared to Figs. 4, 6 and 7. The SEM is 

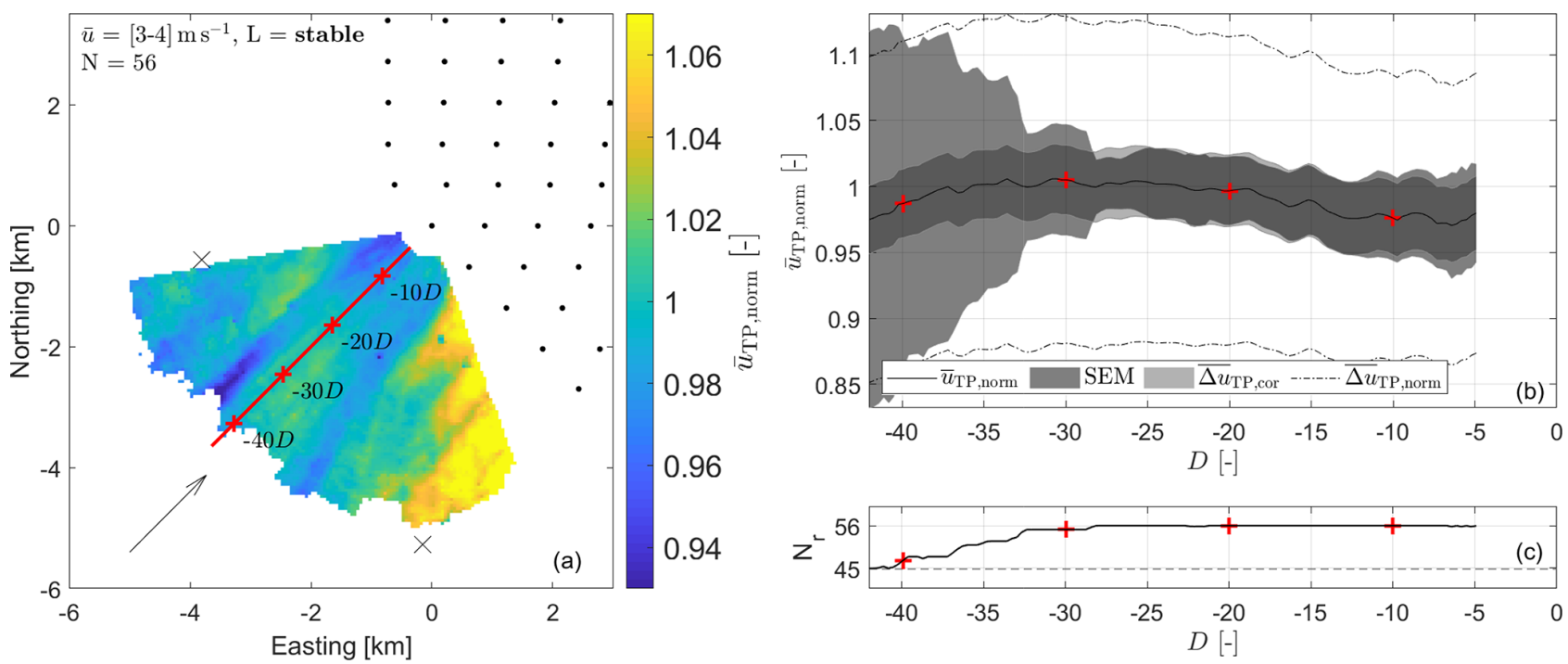

Figure 5. Scenario 2: analysis of lidar scans during stable atmospheric conditions and in cases with a low thrust coefficient (turbines not operating) with $\bar{u}=[3-4] \mathrm{m} \mathrm{s}^{-1}, \chi=[220-230]^{\circ}, N=56, \bar{\chi}=225^{\circ}, \bar{u}_{\mathrm{GT} 58}=3.48 \mathrm{~s}^{-1}, \bar{P}_{\mathrm{GT} 58}=0.06 \mathrm{MW}, L_{\mathrm{med}}=54 \mathrm{~m}$ and $\sigma_{\chi}=2.84^{\circ}$. For details on (a-c) refer to the caption of Fig. 4. Be aware that the SEM here overlays $\overline{\Delta u}_{\mathrm{TP}, \mathrm{cor}}$.
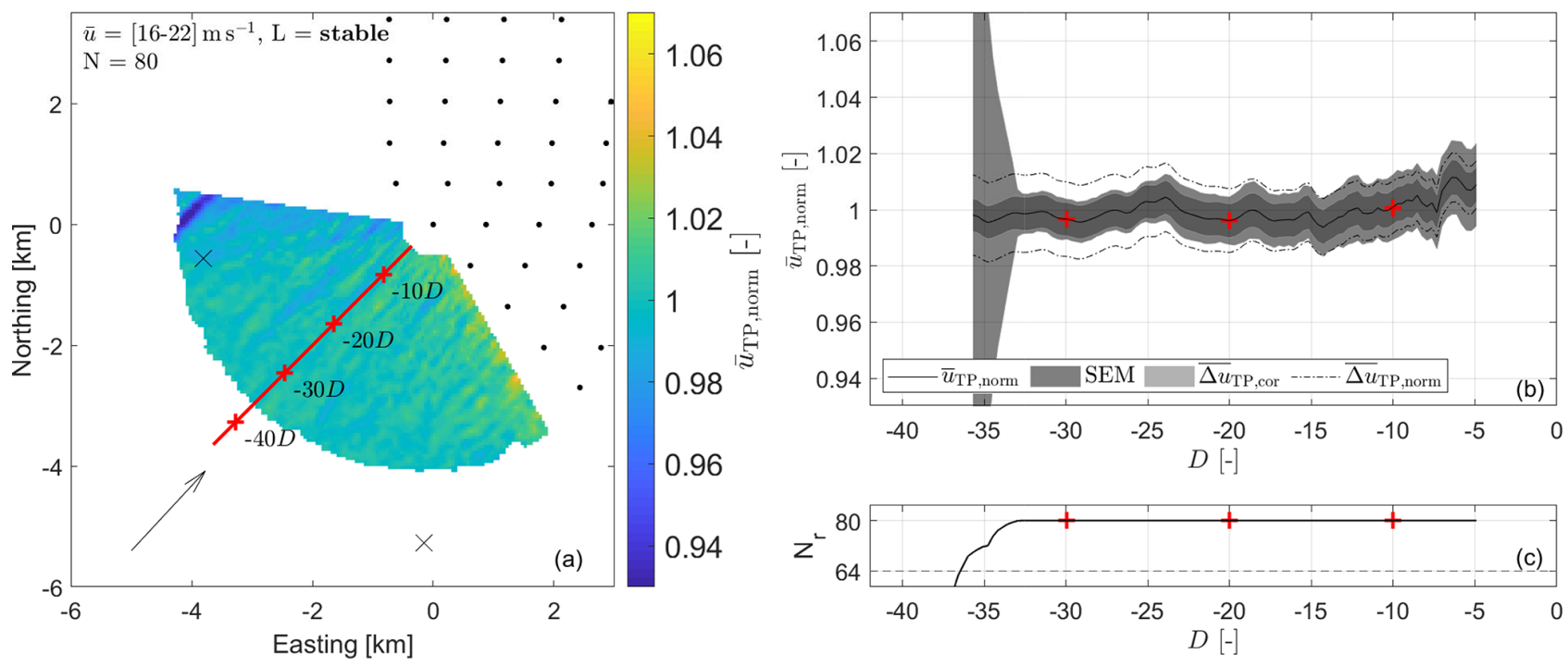

Figure 6. Scenario 3: analysis of lidar scans during stable atmospheric conditions and in cases with a low thrust coefficient with $\bar{u}=[16-$ 22] $\mathrm{m} \mathrm{s}^{-1}, \chi=[220-230]^{\circ}, N=80, \bar{\chi}=223^{\circ}, \bar{u}_{\mathrm{TP}}=19.83 \mathrm{~m} \mathrm{~s}^{-1}, \bar{P}_{\mathrm{GT} 58}=4.79 \mathrm{MW}, L_{\text {med }}=460 \mathrm{~m}$ and $\sigma_{\chi}=1.54^{\circ}$. For details on $(\mathbf{a}-\mathbf{c})$ refer to the caption of Fig. 4 . Be aware that the SEM here overlays $\overline{\Delta u}_{\text {TP,cor }}$.

of a similar size as $\overline{\Delta u}_{\mathrm{TP}, \mathrm{cor}}$; however, it strongly increases with decreasing $N_{\mathrm{r}}$ for increasing distances to the wind farm (Fig. 5c), exceeding $\overline{\Delta u}_{\mathrm{TP}, \text { cor }}$ and finally $\overline{\Delta u}_{\mathrm{TP}, \text { norm }}$ for distances larger than $-40 \mathrm{D}$.

All shown uncertainty ranges are able to account for the observed variations in wind speed. Taking into account also its aforementioned low absolute values, we consider these variations to be insignificant.

\subsection{Scenario 3: wind farm operating above rated wind speed at stable atmospheric conditions with a low thrust coefficient}

Figure 6 visualizes the results of Scenario 3, considering stable atmospheric conditions and the wind farm running far beyond rated wind speed, i.e. at a low thrust coefficient of $\leq 0.3$. No decrease of wind speed close to the wind farm as an indication of global blockage can be observed with $\bar{u}_{\text {TP,norm }}$ fluctuating around a value of 1. As shown in Fig. 6b, similarly as for Scenario 1, the SEM exceeds $\overline{\Delta u}_{\mathrm{TP}, \text { cor }}$ and increases 

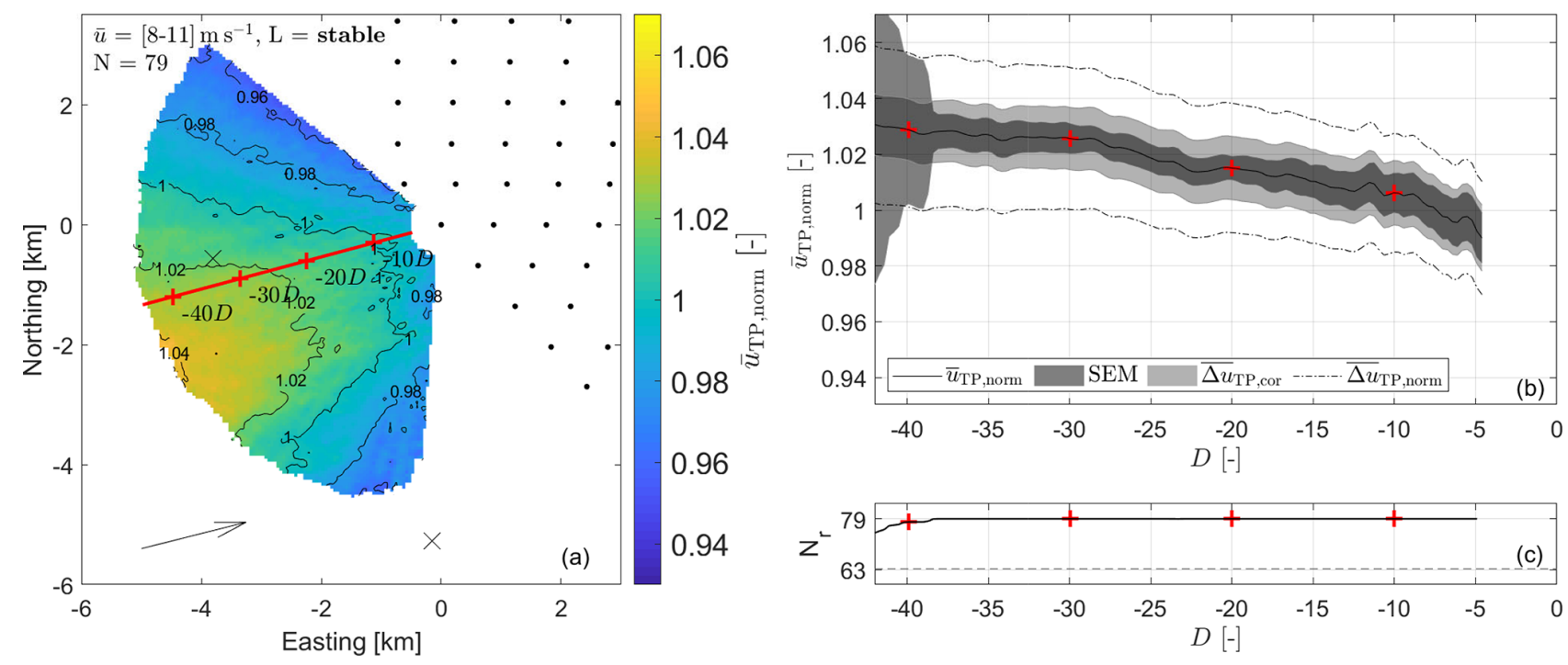

Figure 7. Scenario 4: analysis of lidar scans during stable atmospheric conditions and in cases with a high thrust coefficient with $\bar{u}=[8-$ 11] $\mathrm{m} \mathrm{s}^{-1}, \chi=[250-260]^{\circ}, N=79, \bar{\chi}=256^{\circ}, \bar{u}_{\mathrm{GT} 58}=9.23 \mathrm{~m} \mathrm{~s}^{-1}, \bar{P}_{\mathrm{GT} 58}=4.51 \mathrm{MW}, L_{\text {med }}=307 \mathrm{~m}$ and $\sigma_{\chi}=2.78^{\circ}$. Contour lines in the flow field in (a) highlight the shape of the wind field upstream GT I. For further details on (a-c) refer to the caption of Fig. 4.

strongly for far distances due to decreasing $N_{\mathrm{r}}$ (see Fig. 6c) and decreasing data quality for far range gates. The difference between $\overline{\Delta u}_{\text {TP,cor }}$ and $\overline{\Delta u}_{\text {TP,norm }}$ increases with distance to the wind farm as a consequence of the increasing measuring altitudes and the shape of the wind profile. Also considering the larger mean wind speed, the impact of the uncertainties of $L$ and $z_{\mathrm{m}}$ is here stronger as compared to the unstable cases in Scenario 1.

\subsection{Scenario 4: wind farm operating below rated wind speed at stable atmospheric conditions with a high thrust coefficient}

In Fig. 7 we illustrate the results of Scenario 4, i.e. stable atmospheric conditions and the wind farm operating at a high thrust coefficient. The normalized and averaged wind field shown in Fig. 7a suggests a decrease in wind speed for flow approaching the wind farm. Contour lines highlight the shape of the decrease and show that it is less distinct at the sides of the lidar scan. Observed values of $\bar{u}_{\text {TP,norm }}$ vary between approximately 0.96 and 1.04 . The virtual cut on the wind field given in Fig. $7 \mathrm{~b}$ supports these findings. Starting at a value of about 1.03 at $-44 D$ the normalized and averaged wind speed slowly decreases until it reaches a value of 0.99 at $-5 \mathrm{D}$. The magnitude of the curve's slope hereby increases with decreasing distance to the wind farm. The SEM is narrow compared to $\overline{\Delta u}_{\mathrm{TP}}$,cor and especially $\overline{\Delta u}_{\mathrm{TP} \text {,norm }}$. Only for far distances it strongly increases as a consequence of reduced sample size (see Fig. 7c) and data quality. The total propagated uncertainty $\overline{\Delta u}$ TP,norm reaches values of up to $3 \%$. As the analysed scans are attributed to stable atmospheric conditions, the impact of $L$ and also $z_{\mathrm{m}}$ is large.
As defined in Sect. 2.5, the width of the uncertainty contributions are considered to cover $95 \%$ of all cases. Figure $7 b$ indicates a significant decrease of wind speed closer to the wind farm when considering the corrected propagated uncertainty $\overline{\Delta u}_{\mathrm{TP}, \mathrm{cor}}$. This is not true anymore when including all error contributions, i.e. considering the width of the total propagated uncertainty $\overline{\Delta u}_{\text {TP,norm. }}$. In Fig. 8 we visualize how the decrease of wind speed changes when assuming the largest uncertainties for $L$ and $z_{\mathrm{m}}$ to analyse the error contributions of the range-gate-correlated variables in more detail. We consider the same data set in Fig. 8 as in Fig. 7. Here, we show the two most extreme scenarios with $L-\Delta L, z_{\mathrm{m}}+\Delta z_{\mathrm{m}}$ (blue, largest reducing effect on the deficit) and $L+\Delta L, z_{\mathrm{m}}-\Delta z_{\mathrm{m}}$ (red, largest enhancing effect on the deficit), respectively. As explained earlier, we assume $\Delta L$ and $\Delta z_{\mathrm{m}}$ to be correlated across range gates within the same scan and thus consider the corrected propagated uncertainty $\overline{\Delta u}_{\text {TP,cor }}$ more valuable than the total propagated uncertainty $\overline{\Delta u}_{\text {TP,norm }}$ depicted in Fig. $7 \mathrm{~b}$. As clearly visible in Figs. $7 \mathrm{~b}$ and 8 , misestimations of the Obukhov length and measurement height have a significant impact on the magnitude and shape of the observed wind speed decrease. In the blue graph in Fig. 8 the wind speed deficit is reduced as a consequence of the more stable conditions and larger differences between measuring height and hub height assumed here. Considering the associated uncertainty, the observed wind speed deficit of approximately $2 \%$ for this case with the largest reducing effect tends to be within the range of the corrected propagated uncertainty. When considering errors with less reducing effect, i.e. errors with the same sign but smaller magnitude, the wind speed deficit increases towards a significant value. If maximal errors occur in the opposite di- 


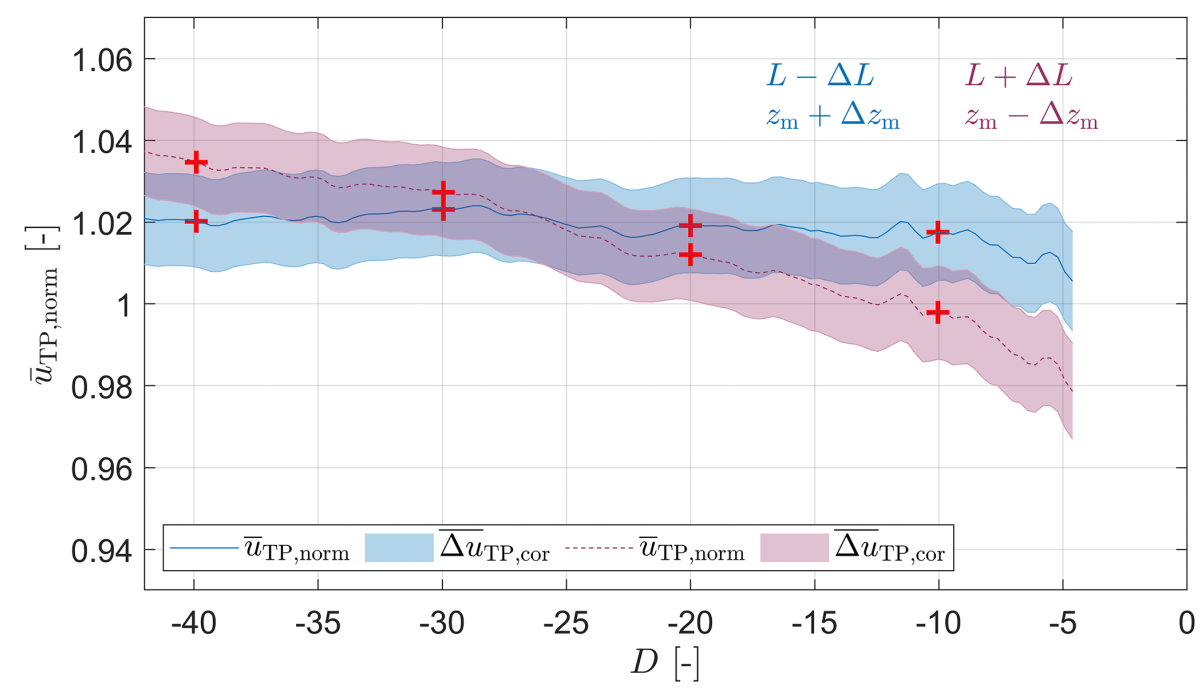

Figure 8. Scenario 4: $\bar{u}_{\text {TP,norm }}$ over the distance to the lidar given in terms of rotor diameter $D$ along the wind field cut, indicated as a red line in Fig. 7a, with the corrected propagated uncertainty $\overline{\Delta u}$ TP,cor visualized as the colour-shaded area. For the blue curve $L$ was additionally reduced by $\Delta L$, and $z_{\mathrm{m}}$ was increased by $\Delta z_{\mathrm{m}}$; for the red curve $L$ was increased, and $z_{\mathrm{m}}$ was reduced, respectively. These cases represent the two combinations of $\Delta L$ and $\Delta z_{\mathrm{m}}$ that yield the most extreme results.

rection (red curve), the effect would be maximally enhanced to a wind speed decrease of $6 \%$. Here, the observed decrease is large compared to the uncertainty intervals and thus clearly significant. Considering the range-gate-correlated error contributions the wind speed deficit of $4 \%$ lies within an uncertainty interval between $2 \%$ and $6 \%$.

Although we only show one wind direction sector, we observed similar wind speed deficits for different sectors. Here we show the most distinct case.

\section{Discussion}

We analysed averaged long-range Doppler lidar PPI scans at TP height in the inflow of the $400 \mathrm{MW}$ offshore wind farm GT I and found wind speed deficits upstream in stably stratified boundary layers with wind turbines operating at a high thrust coefficient in the upper partial load range. In contrast, at unstable stratification and similar operating conditions, no effect was visible. We identified the comparably small wind speed difference by performing a data correction and by averaging the normalized lidar scans. We analysed the effect considering a detailed uncertainty estimation. In this section, we discuss our findings and relate them to the global-blockage effect dependent on atmospheric stability and to the wind farm's operational state as well as possibilities and difficulties for global-blockage measurements.

\subsection{Global blockage dependent on atmospheric and operational conditions}

To distinguish between different wind turbine operational states and atmospheric stabilities, we divided our measurement data into four different scenarios (cf. Sect. 2.4).

Scenario 1. In unstable conditions with wind speeds from 8 to $11 \mathrm{~m} \mathrm{~s}^{-1}$ and a high thrust coefficient (Scenario 1, Fig. 4) we could not identify decreasing wind speeds in front of the wind farm and thus no global-blockage effect. This result is plausible, since wind speed fluctuations in unstable flows are much higher due to convection than the assumed magnitude of global blockage. Convection leads to more mixing in the boundary layer and thus repeals global blockage due to vertical transport of momentum. Furthermore, in unstable stratification, the boundary layer is typically higher, and thus the flow can pass obstacles like hills (Stull, 1988) or in this case a wind farm more easily. The effect of atmospheric stability could be investigated further using a high-fidelity simulation like LES in future studies.

Additionally to Scenario 1 we performed the analysis for unstable stratification and the wind speed ranges above the rated wind speed and below the cut-in wind speed, respectively (cf. Sect. 2.4). In both cases, we could not identify decreasing wind speeds in the inflow of the wind farm. As explained earlier we do not show these results here for brevity.

Scenario 2 and Scenario 3. In stable atmospheric stratification and with low wind turbine thrust coefficients due to low wind speeds (i.e. not operating turbines, Scenario 2, cf. Fig. 5) or due to high wind speeds (i.e. turbines operating with pitched blades above rated wind speed, Scenario 3, cf. Fig. 6) no wind speed reductions upstream of the wind 
farm were identifiable. When the turbines are out of operation there should not be any reason for global blockage to appear due to the very low thrust. For turbines operating above rated wind speed a small global-blockage effect might occur. However, it is unlikely that the effect would be clearly visible in the data as a consequence of high wind speeds and the reduced thrust. Since the turbines operate at rated power, global blockage, if any, would not have a negative impact on power production in this wind speed range.

Scenario 4. In stable atmospheric conditions with a high wind turbine thrust coefficient (i.e. wind turbines operating in the partial load range, Scenario 4, cf. Fig. 7) we found the wind speed to decrease towards the wind farm by approx. $4 \%$ over a distance of $25 D=2.9 \mathrm{~km}$. For larger distances upstream, the wind speed approaches an almost constant value with no further increase visible. We assume the wind speed at $40 \mathrm{D}$ upstream to be the free-stream speed but can not be sure whether small wind-farm-induced effects reach even further. The wind speed reduction is significant when considering range-gate-uncorrelated uncertainties. It is considered meaningful for global blockage to be most significant in stable stratification and for higher thrust coefficients.

Is the observed wind speed deficit global blockage? Despite the intensive uncertainty analysis and error correction we performed in this work (cf. Sect. 2.5), how certain can we be that our observations are caused by the global-blockage effect? To consider the effect of the correlated error sources that we excluded from the calculation of the total propagated uncertainty, namely $\Delta L$ and $\Delta z_{\mathrm{m}}$, Fig. 8 shows the two most extreme cases with the largest reducing effect (blue) and the largest enhancing effect (red) of both error values. Assigning these combinations of errors, the extent of the globalblockage wind speed deficit is limited by $2 \%$ to $6 \%$. In the latter case, the wind speed deficit is clearly significant, while for the first one, it could be explained by the correlated propagated uncertainty. However, considering more likely error magnitudes in between those two most extreme cases, the wind speed deficit would become significant. Thus, we consider the wind speed deficit in front of the wind farm to be caused by the global-blockage effect.

Spatial inhomogeneities in the wind field. Different from wind tunnel measurements where all background conditions could be controlled, free-field measurements cover numerous superimposing meteorological effects. In our case especially the background wind field without the presence of wind farms needs consideration. The small flow effects we observe here are in the order of magnitude as typical wind field fluctuations locally (background turbulence) and over distances of some kilometres (spatial wind field variations). In a single lidar scan it would be not clear whether a wind speed gradient in front of a wind farm is caused by the globalblockage effect or by a spatial variation in the background wind field. Our method of using the average wind field of lots of lidar scans from different days allows us to average different spatial wind speed gradients of the background flow. In
Scenario 1, Scenario 2 and Scenario 3 we found spatial wind speed gradients that are almost zero on average. Scenario 4 shows a negative wind speed gradient. We assume this to be caused mainly by global blockage with the background turbulence and the spatial variation averaged out.

The effect of measurement height. Our measurements of global blockage were performed at a height of approx. $9 \mathrm{~m}$ below the rotor area, while Bleeg et al. (2018) used mainly measurements at hub height and some at $70 \%$ of the hub height. An extrapolation to hub height instead of lidar height would not have a significant impact on our findings, as it would only result in an upscaling of the observed effect to a higher altitude. Further, we assume extrapolation uncertainties would increase significantly when extrapolating across larger height differences (Theuer et al., 2020a). We do not know the vertical distribution of the global-blockage effect but expect it to be equally distributed between the surface and upper-blade-tip height. Bleeg et al. (2018) found only small variations in the analysed blockage effect comparing mast measurements at hub height and $70 \%$ of the hub height. Computational fluid dynamics (CFD) results indicate a rather constant global-blockage effect up to at least hub height due to the presence of the ground (Branlard et al., 2020). In this investigation, we did not study global-blockage-induced flow deflections upwards, downwards or sideways which could lead to increased wind speeds above, below or beside the wind farm's rotor area. The vertical extent of the globalblockage effect in front of a wind farm needs to be assessed in future experimental studies to verify numerical results.

Influences of cluster wakes. Schneemann et al. (2020) show the existence of cluster wakes in the inflow of GT I using data from the same measurement campaign as used here. In the wind directions we chose for the analysis of global blockage, no distinct wind speed gradients are present in the inflow. Schneemann et al. (2020) did not find signatures of the wakes of single wind turbines in the inflow of GT I. Nygaard and Newcombe (2018) showed dual-Doppler radar measurements of a wind farm wake with the signatures of single wind turbines disappearing less than $10 \mathrm{~km}$ behind the farm. Generally, the data we present here could be influenced by cluster wakes. However, we do not expect disturbances of the global-blockage measurement, since the centre flow of a cluster wake in the far field is comparably homogeneous and would only reduce the mean wind speed in the whole lidar scan. Recovery of the possible cluster wake influence with a positive wind speed gradient towards GT I could on the other hand contradict the global-blockage effect with a negative wind speed gradient. This would lead to a reduction of the observable global-blockage effect. Furthermore, cluster wakes could have an influence on the prevailing wind profile that can not be quantified here. This is the subject of current research.

The magnitude of global blockage. We found the magnitude of the global-blockage-induced deficit of approx. $4 \%$ (uncertainty range of $2 \%$ to $6 \%$ ) in stable stratification to 
correspond well with values measured in an onshore freefield experiment by Bleeg et al. (2018) based on met mast point measurements at three different wind farms of typically $2 \%$ to $4 \%$. One possible explanation of the comparably lower deficits of Bleeg et al. (2018) is the lack of stability information and thus the comparison of long intervals including the climate mean of stratifications. Our results suggest less or no global-blockage effects in unstable stratification; this effect possibly reduced the average values of Bleeg et al. (2018). RANS simulations performed by Bleeg et al. (2018) typically showed similar or slightly smaller global-blockage deficits. Wu and Porté-Agel (2017) found global-blockage deficits in LES simulations of large finite-size wind farms of $1.3 \%$ and $3 \%$ for different farm layouts in a weak free atmosphere with neutral stratification across the rotor area, which is slightly lower than our findings. When discussing the strength of global blockage in our data, we need to consider the measurement distances. We analysed a wind speed difference between 40 and $4 D$ upstream. At this far distance the effect seems to have almost vanished with a constant wind speed. Nevertheless, a further slight increase in wind speed for larger distances is possible. Moreover, the strong wind speed gradients at the lower distance of $4 D$ suggest an even further wind speed decrease towards the wind farm. Therefore, we assume the global-blockage effect to be even stronger than quantified here.

The spatial extent of global blockage. Besides results from wind tunnel experiments (Segalini and Dahlberg, 2019) and onshore free-field point measurements (Bleeg et al., 2018) our lidar measurements represent the first areal free-field investigation of the global-blockage effect offshore. Spatial analysis of global blockage has only been reported from numerical studies so far. RANS simulations performed by Bleeg et al. (2018) for three different large onshore wind farms reveal homogeneous induction zones upstream of the farms with spatial extents of more than $2 \mathrm{~km}$ for a deficit of $1 \%$. Such distances correspond well to our findings in Scenario 4 . The higher wind speed deficits in our data could possibly be explained by the restriction to stable stratification. Nevertheless, the shape of the induction zones in their RANS simulations seem to smoothly follow the first row of turbines. The contours we show in Fig. 7 tend to have the same shape in the middle sector of the wind field but deviate from that shape on the sides. We assume this behaviour to be mainly related to the applied assumption of a homogeneous wind direction. With an increasing angular difference of the lidar's azimuth angle from the wind direction the orthogonal components of the main wind direction, i.e. local deviations in the wind direction, increasingly contaminate the measurement of the local horizontal wind under the assumption of a homogeneous wind direction (cf. Sect. 2.5.4). Flow effects resulting from the wind farm's blockage with wind components tending to flow around the wind farm (Porté-Agel et al., 2019) can influence the observed contours. The exemplary local deviation of the estimated horizontal wind field we show in Fig. 3 assumes a diverging flow with wind direction deviations of $2.0^{\circ}$. This low value of divergence could well explain the observed shape of the contours with underestimated horizontal wind speeds of more than $4 \%$. Future experimental studies should focus on assessing the globalblockage-induced flow around the farm from dual-Doppler lidar measurements or single-lidar measurements with more advanced analysis techniques.

The influence of global blockage on power production. To assess the impact of the global-blockage effect on a wind farm's annual energy production (AEP) more research and development on the implementation and validation of the effect in wind farm planning tools is needed. A detailed AEP assessment then needs to consider particularly the local undisturbed wind speed and stability wind roses. The consideration of global blockage in the future could further increase the accuracy of wind energy site assessment, which is especially important for the financing process of wind farm projects. Despite its possible negative impact on energy production, global blockage seems not to have a critical impact on wind energy utilization. In our study, we observed the effect only below rated power, in stable stratification and with a magnitude of $4 \%$ within the uncertainty range of $2 \%$ to $6 \%$. Consequently, we expect global blockage to have a much lower impact on the power production than other wind farm flow features like inner-wind-farm wakes.

\subsection{Global-blockage measurement techniques}

Compared to wind turbine wake effects with a wind speed deficit of several metres per second over a distance of less than one rotor radius between wake centre and free flow, the global-blockage effect has a comparably small magnitude. Scenario 4 reveals a deficit of about $4 \%$ of the average wind speed of $9.2 \mathrm{~m} \mathrm{~s}^{-1}$, which equals approx. $0.4 \mathrm{~m} \mathrm{~s}^{-1}$. This difference builds up over a distance of $25 D=2.9 \mathrm{~km}$. This is well below typical fluctuations in wind fields due to e.g. orographic or thermal influences which makes global blockage hard to identify in measurement data.

There are no further areal wind field measurements of global blockage in the literature. The shape of the zone with reduced wind speeds in front of the wind farm and comparisons of different locations could not be analysed using single-point measurements like those presented by Bleeg et al. (2018). Spatial characteristics of global-blockage inflows of wind farms that were generated by numerical simulations and modelling (Bleeg et al., 2018; Branlard and Meyer Forsting, 2020) have not been experimentally verified, yet. Different from point measurements areal lidar wind field measurements of the wind farm inflow resolving the zone with wind speed reduction can allow for a more detailed analysis.

Generally, we do not expect global blockage to be significantly identifiable in single flow measurements like an individual lidar scan. The effect is much smaller than the com- 
mon fluctuations in wind farm inflows and needs to be derived from averaged measurements where the influence of local turbulence and coherent turbulent structures is reduced in the averaging process.

The lidar measurements we analyse in this paper were originally performed to study the effect of cluster wakes in the inflow of GT I (Schneemann et al., 2020) and to perform minute-scale power forecasts (Theuer et al., 2020b). Due to the comparably small global-blockage effect, all errors influencing the accuracy of lidar measurements need to be carefully examined and reduced wherever possible. We give an overview on sources of uncertainty in Table 2. For lidar measurement campaigns aiming at the assessment of global blockage or similar small flow effects we recommend doing the following.

- Calibrate the lidar before the campaign. This includes the measurement of radial velocities, the range gate distance from the device and especially the scanner orientation and movements. Here especially the scanner's elevation angle deviation is crucial, since it results in height errors of the measurement.

- Carefully align the lidar at the measurement location and monitor the lidar's tilt dynamically. We recommend using accurate inclinometers and accelerometers and in offshore campaigns the method of "sea surface levelling" for lidar tilt alignment and the method of "hard targeting" for alignment of the north direction (Rott et al., 2017, 2021).

- Perform independent measurements of the prevailing wind profile either by e.g. met mast, VAD lidar or virtual met masts spanned by scanning lidars (Bell et al., 2020) to be able to perform a proper height correction of the scanning lidar data.

- Perform measurements of meteorological quantities for characterization of atmospheric stability to support a more precise interpolation of the wind profile (e.g. Schneemann et al., 2020).

The stronger tilting on the nacelle compared to the transition piece and the resulting large errors in the measurement height introduce increased uncertainties to nacellebased measurements especially when aiming to achieve several kilometres of range or to detect small flow effects like global blockage. Active motion compensation of the lidar's scanner or similar measures could enable the possibility of nacelle-based measurements.

Further, the use of overlapping dual-Doppler measurements could be beneficial to better resolve local flow characteristics like global-blockage-induced flow deflections and to overcome the need for basic assumptions like the homogeneity of the wind field in the VAD algorithm (e.g. van Dooren et al., 2016; Stawiarski et al., 2013). Another measurement system to assess global blockage could be the remote sensing method Doppler radar which was successfully deployed for wind turbine and wind farm wake measurements (Nygaard and Newcombe, 2018).

\section{Conclusions}

This paper has pursued the objective of analysing whether it is possible to measure global wind farm blockage with longrange Doppler lidar dependent on different atmosphericstability estimates and wind farm operational states. We present averaged lidar PPI measurements of the inflow of the 400 MW offshore wind farm Global Tech I. In stable stratification and with the turbines operating below and up to rated power with a high thrust coefficient, the measurements revealed reduced wind speeds at the height of the transition piece in the approaching flow. In unstable stratification and similar operating conditions, however, no effect was visible. We relate this upstream wind speed reduction to the presence of the wind farm, namely to global wind farm blockage. Therefore, we conclude global blockage to be dependent on atmospheric stability.

Compared to wind turbine wakes or cluster wakes, global blockage is a very small effect that is overlaid with different atmospheric phenomena and thus very hard to detect. Nevertheless, based on our detailed uncertainty assessment we arrive at the conclusion that the wind speed deficit in front of Global Tech I in our lidar measurements is caused by global blockage. Generally, we assume long-range Doppler lidar to be able to accurately measure global blockage and recommend carefully aligning and calibrating the used lidar systems.

Our measurements agree with recent findings of the magnitude of the global-blockage effect to range from $2 \%$ to $6 \%$. At the platform level, we found a wind speed reduction of $4 \%$ within an uncertainty range from $2 \%$ to $6 \%$, over a distance of approx. $2.9 \mathrm{~km}$ or $25 \mathrm{D}$. The influence of the global-blockage effect on the annual energy production of a wind farm requires further experimental and numerical investigations. Due to the expected limited appearance of global blockage only in special atmospheric situations and wind farm operational states and its small magnitude, we expect the impact on power production to be much smaller in comparison to inner-wind-farm wakes. Accurate estimates of the global-blockage effect by means of well-calibrated engineering models could further decrease uncertainties in wind farm site assessments and power calculations in the future.

In this work, we demonstrated scanning long-range Doppler lidar to be a suitable tool to study global wind farm blockage and provide strong evidence for the existence of the global-blockage effect for a wind farm with the turbines operating at high thrust coefficients in a stably stratified atmosphere. 
Data availability. Lidar and meteorological data are not published and could be made available on request. The OSTIA data set can be obtained from http://marine.copernicus.eu (Copernicus marine service, 2021). A set of GT I wind turbine coordinates can be found at https://www.openstreetmap.org (OpenStreetMap, 2021).

Author contributions. JS initiated the research; performed the measurement campaign; was heavily involved in the funding acquisition and the research discussion; wrote Sects. 1, 2.1, 2.2, 2.5.4, 4 and 5; and prepared Figs. 1, 2 and 3. FT performed the data analysis; was heavily involved in the research discussion; wrote Sects. 2.3, 2.4, 2.5 and 3; and made Figs. 4, 5, 6, 7 and 8. AR was heavily involved in the research discussion and supported the writing of the paper. MD initiated the research and was heavily involved in the funding acquisition and the research discussion. MK was heavily involved in the funding acquisition and supervised the research. All authors contributed intensively to an internal review.

Competing interests. The authors declare that they have no conflict of interest.

Acknowledgements. We acknowledge the wind farm operator Global Tech I Offshore Wind GmbH for providing SCADA data as well as their support of the work and the measurement campaign. Thanks go to the Met Office for making the OSTIA data set available. Special thanks go to Stephan Voß, ForWind Oldenburg, for his great support in conducting of the measurement campaign and to Julia Gottschall, Fraunhofer IWES, for an interesting discussion and helpful comments.

Financial support. This research has been supported by the Bundesministerium für Wirtschaft und Energie in the framework of the research projects "OWP Control" (grant no. FKZ 0324131A) and "X-Wakes" (grant nos. FKZ 03EE3008A and D) on the basis of a decision by the German Bundestag. Frauke Theuer is supported by the Deutsche Bundesstiftung Umwelt (grant no. 20018/582).

Review statement. This paper was edited by Julie Lundquist and reviewed by James Bleeg, Emmanuel Branlard, and Greg Poulos.

\section{References}

Allaerts, D. and Meyers, J.: Gravity Waves and Wind-Farm Efficiency in Neutral and Stable Conditions, Bound.-Lay. Meteorol., 166, 269-299, https://doi.org/10.1007/s10546-017-0307-5, 2017.

Bell, T. M., Klein, P., Wildmann, N., and Menke, R.: Analysis of flow in complex terrain using multi-Doppler lidar retrievals, Atmos. Meas. Tech., 13, 1357-1371, https://doi.org/10.5194/amt13-1357-2020, 2020.

Bleeg, J.: A Graph Neural Network Surrogate Model for the Prediction of Turbine Interaction Loss, J. Phys. Conf. Ser., 1618,
062054, https://doi.org/10.1088/1742-6596/1618/6/062054, 2020.

Bleeg, J., Purcell, M., Ruisi, R., and Traiger, E.: Wind Farm Blockage and the Consequences of Neglecting Its Impact on Energy Production, Energies, 11, 1609, https://doi.org/10.3390/en11061609, 2018.

Branlard, E. and Meyer Forsting, A. R.: Assessing the blockage effect of wind turbines and wind farms using an analytical vortex model, Wind Energy, 23, 2068-2086, https://doi.org/10.1002/we.2546, 2020.

Branlard, E., Quon, E., Meyer Forsting, A. R., King, J., and Moriarty, P.: Wind farm blockage effects: comparison of different engineering models, J. Phys. Conf. Ser., 1618, 062036, https://doi.org/10.1088/1742-6596/1618/6/062036, 2020.

Copernicus marine service: Copernicus Marine environment monitoring service, available at: http://marine.copernicus.eu/, last access: 28 March 2021.

Dyer, A. J.: A review of flux-profile relationships, Bound.-Lay. Meteorol., 7, 363-372, https://doi.org/10.1007/BF00240838, 1974.

Emeis, S.: Wind Energy Meteorology, 2nd edition, Springer International Publishing AG, https://doi.org/10.1007/978-3-31972859-9, 2018.

EnBW: EnBW Hohe See and Albatros wind farms, The construction diary for Hohe See, online, available at: https://www.enbw.com/renewable-energy/wind-energy/ our-offshore-wind-farms/hohe-see/construction-diary.html (last access 28 March 2021), 2019.

Global Tech I Offshore Wind GmbH: Global Tech I, online, available at: https://globaltechone.de/power/ (last access: 5 January 2021), 2021.

Good, S., Fiedler, E., Mao, C., Martin, M. J., Maycock, A., Reid, R., Roberts-Jones, J., Searle, T., Waters, J., While, J., and Worsfold, M.: The Current Configuration of the OSTIA System for Operational Production of Foundation Sea Surface Temperature and Ice Concentration Analyses, Remote Sens.-Basel, 12, 720, https://doi.org/10.3390/rs12040720, 2020.

Hasager, C. and Sjöholm, M.: Editorial for the Special Issue "Remote Sensing of Atmospheric Conditions for Wind Energy Applications", Remote Sens.-Basel, 11, 781, https://doi.org/10.3390/rs11070781, 2019.

Högström, U.: Non-Dimensional Wind and Temperature Profiles in the Atmospheric Surface Layer: A Re-Evaluation, Bound.Lay. Meteorol., 42, 55-78, https://doi.org/10.1007/BF00119875, 1988.

IEC: IEC 61400-12 Wind turbine generator systems - Part 12: Wind turbine power performance testing, International Electrotechnical Commission, Geneva, Switzerland, 2017.

Kitaigorodskii, S. A. and Joffre, S. M.: In search of a simple scaling for the height of the stratified atmospheric boundary layer, Tellus A, 40, 419-433, https://doi.org/10.3402/tellusa.v40i5.11812, 1988.

Käsler, Y., Rahm, S., Simmet, R., and Kühn, M.: Wake Measurements of a Multi-MW Wind Turbine with Coherent Long-Range Pulsed Doppler Wind Lidar, J. Atmos. Ocean. Tech., 27, 15291532, https://doi.org/10.1175/2010JTECHA1483.1, 2010.

McKillup, S.: Statistics Explained: An Introductory Guide for Life Scientists, chap. 6 - Working from samples data, populations and statistics, Cambridge University Press, https://doi.org/10.1017/CBO9780511815935, pp. 57-76, 2005. 
Menke, R., Vasiljević, N., Wagner, J., Oncley, S. P., and Mann, J.: Multi-lidar wind resource mapping in complex terrain, Wind Energ. Sci., 5, 1059-1073, https://doi.org/10.5194/wes-5-10592020, 2020.

Meyer Forsting, A. R., Troldborg, N., and Gaunaa, M.: The flow upstream of a row of aligned wind turbine rotors and its effect on power production, Wind Energy, 20, 63-77, https://doi.org/10.1002/we.1991, 2016.

Meyer Forsting, A. R., Troldborg, N., Bechmann, A., and Réthoré, P.-E.: Modelling Wind Turbine Inflow: The Induction Zone, $\mathrm{PhD}$ thesis, DTU Wind Energy, https://doi.org/10.11581/DTU:00000022, 2017.

Møller, M., Domagalski, P., and Sætran, L. R.: Comparing abnormalities in onshore and offshore vertical wind profiles, Wind Energ. Sci., 5, 391-411, https://doi.org/10.5194/wes-5-391-2020, 2020.

Nygaard, N. G. and Newcombe, A. C.: Wake behind an offshore wind farm observed with dual-Doppler radars, J. Phys. Conf. Ser., 1037, 072008, https://doi.org/10.1088/17426596/1037/7/072008, 2018.

Nygaard, N. G., Steen, S. T., Poulsen, L., and Pedersen, J. G.: Modelling cluster wakes and wind farm blockage, J. Phys. Conf. Ser., 1618, 062072, https://doi.org/10.1088/17426596/1618/6/062072, 2020.

OpenStreetMap Foundation on behalf of the community: OpenStreetMap, available at: https://www.openstreetmap.org/, last access: 28 March 2021.

Ørsted A/S: Ørsted presents update on its long-term financial targets, online, available at: https://orsted.com/en/ Company-Announcement-List/2019/10/1937002/ (last access: 28 March 2021), 2019.

Peña, A., Gryning, S.-E., and Hasager, C. B.: Measurements and Modellin of the Wind Speed Profile in the Marine Atmospheric Boundary Layer, Bound.-Lay. Meteorol., 129, 479-495, https://doi.org/10.1007/s10546-008-9323-9, 2008.

Porté-Agel, F., Bastankhah, M., and Shamsoddin, S.: Wind-Turbine and Wind-Farm Flows: A Review, Bound.-Lay. Meteorol., 174, 1-59, https://doi.org/10.1007/s10546-019-00473-0, 2019.

Rodrigo, J. S., Cantero, E., García, B., Borbón, F., Irigoyen, U., Lozano, S., Fernande, P. M., and Chávez, R. A.: Atmospheric stability assessment for the characterization of offshore wind conditions, J. Phys. Conf. Ser., 625, 012044, https://doi.org/10.1088/1742-6596/625/1/012044, 2015.

Rohrig, K., Berkhout, V., Callies, D., Durstewitz, M., Faulstich, S., Hahn, B., Jung, M., Pauscher, L., Seibel, A., Shan, M., Siefert, M., Steffen, J., Collmann, M., Czichon, S., Dörenkämper, M., Gottschall, J., Lange, B., Ruhle, A., Sayer, F., Stoevesandt, B., and Wenske, J.: Powering the 21 st century by wind energy-Options, facts, figures, Appl. Phys. Rev., 6, 031303, https://doi.org/10.1063/1.5089877, 2019.

Rott, A., Schneemann, J., Trabucchi, D., Trujillo, J. J., and Kühn, M.: Accurate deployment of long range scanning lidar on offshore platforms by means of sea surface leveling, in: Poster presentation NAWEA Windtech, Windtech 2017 - International Conference on Future Technologies for Wind Energy, Boulder, Colorado, USA, 24-26 October 2017.
Rott, A., Schneemann, J., Theuer, F., Quintero, J. J. T., and Kühn, M.: Alignment of scanning lidar in offshore wind farms, Atmos. Meas. Tech., in preparation, 2021.

Schneemann, J., Voß, S., Rott, A., and Kühn, M.: Doppler wind lidar plan position indicator scans and atmospheric measurements at the offshore wind farm "Global Tech I", PANGAEA - Data Publisher for Earth \& Environmental Science, https://doi.org/10.1594/PANGAEA.909721, 2019.

Schneemann, J., Rott, A., Dörenkämper, M., Steinfeld, G., and Kühn, M.: Cluster wakes impact on a far-distant offshore wind farm's power, Wind Energ. Sci., 5, 29-49, https://doi.org/10.5194/wes-5-29-2020, 2020.

Segalini, A. and Dahlberg, J.-A.: Blockage effects in wind farms, Wind Energy, 23, 120-128, https://doi.org/10.1002/we.2413, 2019.

Smith, S. D.: Wind Stress and Heat Flux over the Ocean in Gale Force Winds, J. Phys. Oceanogr., 10, 709-726, https://doi.org/10.1175/15200485(1980)010<0709:WSAHFO>2.0.CO;2, 1980.

Stawiarski, C., Träumner, K., Knigge, C., and Calhoun, R.: Scopes and Challenges of Dual-Doppler Lidar Wind Measurements-An Error Analysis, J. Atmos. Ocean. Tech., 30, 2044 2062, https://doi.org/10.1175/JTECH-D-12-00244.1, 2013.

Stull, R. B. (ed.): An Introduction to Boundary Layer Meteorology, Kluwer Academic Publishers, Dordrecht, The Netherlands, https://doi.org/10.1007/978-94-009-3027-8, 1988.

Theuer, F., van Dooren, M. F., von Bremen, L., and Kühn, M.: On the accuracy of a logarithmic extrapolation of the wind speed measured by horizontal lidar scans, J. Phys. Conf. Ser., 1618, 032043, https://doi.org/10.1088/17426596/1618/3/032043, 2020a.

Theuer, F., van Dooren, M. F., von Bremen, L., and Kühn, M.: Minute-scale power forecast of offshore wind turbines using long-range single-Doppler lidar measurements, Wind Energ. Sci., 5, 1449-1468, https://doi.org/10.5194/wes-5-1449-2020, 2020b.

Trabucchi, D., Trujillo, J. J., Schneemann, J., Bitter, M., and Kühn, M.: Application of staring lidars to study the dynamics of wind turbine wakes, Meteorol. Z., 24, 557-564, https://doi.org/10.1127/metz/2014/0610, 2014.

van Dooren, M., Trabucchi, D., and Kühn, M.: A Methodology for the Reconstruction of 2D Horizontal Wind Fields of Wind Turbine Wakes Based on Dual-Doppler Lidar Measurements, Remote Sens.-Basel, 8, 809, https://doi.org/10.3390/rs8100809, 2016.

Werner, C.: Doppler Wind Lidar, in: Lidar, edited by: Weitkamp, C., vol. 102 of Springer Series in Optical Sciences, Springer-Verlag, New York, NY, https://doi.org/10.1007/0-387-25101-4_12, pp. 325-354, 2005.

Wu, K. and Porté-Agel, F.: Flow Adjustment Inside and Around Large Finite-Size Wind Farms, Energies, 10, 2164, https://doi.org/10.3390/en10122164, 2017. 